\title{
An EZH2-dependent transcriptional complex promotes aberrant epithelial remodelling after injury
}

\author{
Huy Q Le 1, $^{\text {(DD, Matthew A Hill }}{ }^{1,2}$, Ines Kollak ${ }^{1}$, Martina Keck ${ }^{1}$, Victoria Schroeder ${ }^{1}$, Johannes Wirth ${ }^{1}$, \\ Wioletta Skronska-Wasek ${ }^{1}$, Eva Schruf ${ }^{1}$, Benjamin Strobel ${ }^{3}$ D , Heiko Stahl ${ }^{1}$, Franziska E Herrmann ${ }^{1}$, \\ Alexandre R Campos ${ }^{4}$, Jun Li ${ }^{5}$, Karsten Quast ${ }^{6}$, Dagmar Knebel ${ }^{6}$, Coralie Viollet ${ }^{6}$, Matthew J Thomas ${ }^{1,2}$, \\ David Lamb ${ }^{1}$ \& James P Garnett ${ }^{1,7}$
}

\begin{abstract}
Unveiling the molecular mechanisms of tissue remodelling following injury is imperative to elucidate its regenerative capacity and aberrant repair in disease. Using different omics approaches, we identified enhancer of zester homolog $2(E Z H 2)$ as a key regulator of fibrosis in injured lung epithelium. Epithelial injury drives an enrichment of nuclear transforming growth factor- $\beta$-activated kinase 1 (TAK1) that mediates EZH2 phosphorylation to facilitate its liberation from polycomb repressive complex 2 (PRC2). This process results in the establishment of a transcriptional complex of EZH2, RNA-polymerase II (POL2) and nuclear actin, which orchestrates aberrant epithelial repair programmes. The liberation of EZH2 from PRC2 is accompanied by an EZH2-EZH1 switch to preserve $\mathrm{H} 3 \mathrm{~K} 27 \mathrm{me} 3$ deposition at non-target genes. Loss of epithelial TAK1, EZH2 or blocking nuclear actin influx attenuates the fibrotic cascade and restores respiratory homeostasis. Accordingly, EZH2 inhibition significantly improves outcomes in a pulmonary fibrosis mouse model. Our results reveal an important noncanonical function of EZH2, paving the way for new therapeutic interventions in fibrotic lung diseases.
\end{abstract}

Keywords EZH2; fibrosis; lung epithelial injury; nuclear actin; TAK1

Subject Categories Chromatin, Transcription \& Genomics; Molecular Biology of Disease; Signal Transduction

DOI 10.15252/embr.202152785 | Received 4 March 2021 | Revised 5 June

2021 | Accepted 10 June 2021

EMBO Reports (2021) e52785

\section{Introduction}

How organisms activate repair and regeneration programmes upon injury to restore physiological conditions remains a fundamental question in biology. This process arises from communication between several cell types to coordinate changes in their gene expression and behaviour that lead to tissue remodelling (Zepp \& Morrisey, 2019). Cells sense and transmit these signals through receptors, cell-cell and cell-extracellular matrix (ECM) interactions that couple extrinsic signals through the actomyosin cytoskeleton to the nucleus and eventually the chromatin. This signalling plays a central role in organ homeostasis and any dysregulation may result in the transition to disease, for example cancer or fibrosis (Sasai, 2013; Wickstrom \& Niessen, 2018).

Idiopathic pulmonary fibrosis (IPF) is a chronic respiratory disease characterised by progressive fibrotic lung remodelling and respiratory failure. The disease is ultimately fatal, despite the emergence of current therapeutics (Hewlett et al, 2018). IPF initiation and progression have been linked to damage and remodelling of the respiratory epithelium through evidence derived from genetic screening, animal models of fibrosis and pathological analyses of patient lungs (Sakai \& Tager, 2013; Zepp \& Morrisey, 2019; Selman \& Pardo, 2020). Although these studies suggest that aberrant epithelial remodelling and their crosstalk with mesenchymal cells (MCs) are key drivers of IPF disease pathology, the underlying mechanisms are still not fully understood (Selman \& Pardo, 2020).

TGF $\beta 1$ is a predominant effector in most forms of fibrosis; it is secreted and tethered to the ECM by many different cell types, including epithelial and MCs (Kim et al, 2018). Previous studies have uncovered an intricate crosstalk between SMADs, the central player of TGF $\beta$ signalling and a myriad of epigenetic regulators that

\footnotetext{
1 Lung Repair \& Regeneration Department, Boehringer Ingelheim Pharma GmbH \& Co. KG, Biberach, Germany

2 University of Bath, Bath, UK

3 Drug Discovery Sciences, Boehringer Ingelheim Pharma GmbH \& Co. KG, Biberach, Germany

4 Sanford Burnham Prebys, Medical Discovery Institute, La Jolla, CA, USA

Immunology and Respiratory Disease Research Department, Boehringer Ingelheim Pharmaceuticals, Inc, Ridgefield, CT, USA

6 Global Computational Biology and Digital Sciences, Boehringer Ingelheim Pharma GmbH \& Co. KG, Biberach, Germany

7 Translational and Clinical Research Institute, Newcastle University, Newcastle, UK

*Corresponding author. Tel: +49 735154 175014; E-mail: quang_huy.le@boehringer-ingelheim.com
} 
fine-tune transcriptional machinery (Andrews et al, 2019; Lu et al, 2019). PRC2 is a major epigenetic repressive regulator consisting of EZH2, SUZ12 and EED. PRC2 catalyses mono-, di- and trimethylation of histone 3 on lysine 27 (H3K27me1/2/3) through its methyltransferase EZH2 subunit resulting in gene silencing and chromatin compaction (Skourti-Stathaki et al, 2019). Importantly, EZH2 overexpression has been detected in fibrosis and cancer, showing a positive correlation with their progression (Grindheim et al, 2019; Tsou et al, 2019). Furthermore, a non-canonical role of EZH2 as a transcriptional activator, marked by phosphorylation on threonine 311 (T311) or T487, has been identified in several cancers (Yan et al, 2016; Wan et al, 2018). Studies in embryonic stem cells have suggested that the transition to non-canonical EZH2 is mediated by the occupancy of poised POL2 on target genes in a permissive chromatin environment that primes enhancer-promoter interactions for future gene activation (Brookes et al, 2012; CruzMolina et al, 2017). However, little is known about the molecular principles dictating the role of EZH2 as a transcriptional silencer or activator in disease states.

Here, we describe for the first time a PRC2-independent role of EZH2 as a key transcriptional regulator driving aberrant repair in injured respiratory epithelial cells. This requires a liberation of EZH2 from the PRC2 complex triggered by TAK1 to establish a fibrotic transcriptional complex of EZH2, POL2 and nuclear actin. Simultaneously, EZH1 is recruited and forms an EZH1-PRC2 complex to maintain the silencing of non-target genes. These changes activate a fibrotic crosstalk with MCs, leading to ECM remodelling and further damage of the epithelium. Characterisation of this pathologic network provides an opportunity for novel therapeutic interventions through inhibition of non-canonical EZH2.

\section{Results}

\section{Injured epithelial cells activate a fibrotic cascade with mesenchymal cells}

To mimic epithelial-mesenchymal crosstalk in the human lung upon injury, we utilised a co-culture system wherein human lung alveolar epithelial cells (AECs; Fernandez \& Eickelberg, 2012) were injured by apical treatment with TGF $\beta 1$ on transwell inserts, followed by co-culture with either primary human lung MCs or IPF-derived lung MCs (IPF-MCs) for $72 \mathrm{~h}$ (Fig 1A). We hypothesised that, in response to TGF $\beta 1$-induced injury, the AECs secrete profibrotic multifactorial stimuli that activate MCs. We therefore purposefully applied the same amount of TGF $\beta 1$ on the apical surface of the transwell in the MCs mono-culture to exclude any direct effect of TGF $\beta 1$ on MCs. Apical TGF $\beta 1$ treatment of AECs led to the loss of E-cadherin (E-cad), a marker for epithelial integrity (Fig 1B and C). This coincided with enhanced alpha smooth muscle actin ( $\alpha \mathrm{SMA}$ ) and other profibrotic/ ECM proteins including fibronectin (FN), type I pro-collagen (pro COL-1), matrix metalloproteinase-7 (MMP7) and monocyte chemotactic protein 1 (MCP-1) in the mesenchymal compartment (Appendix Fig S1A-E). Importantly, this profibrotic response to TGF $\beta 1$-induced injury was only observed in the presence of an epithelial layer; and no significant elevation of TGF $\beta 1$ could be detected in the mesenchymal compartment after $72 \mathrm{~h}$ in the coculture system (Appendix Fig S1F and G). In addition, blocking TGF $\beta 1$ signalling by an ALK5 inhibitor (Roth et al, 2010) in MCs was unable to attenuate the effect of injured AECs on MCs (Appendix Fig S1H), indicating the existence of complex multifactorial signals which drive profibrotic response. Interestingly, this effect was even more pronounced in IPF-derived MCs (Fig 1 D and E and Appendix Fig S1I), suggesting that MCs in the coculture system are capable of retaining long-term memory of their origin associated with a rapid response when exposed to a fibrotic-like signal. To address this point, we examined different MCs and IPF-MC donors either directly with TGF $\beta 1$ or in the coculture system. We observed no changes in response to direct TGF $\beta 1$ treatment between MCs and IPF-MCs. In contrast, IPFMCs in co-culture with injured AECs display higher aSMA levels compared to their healthy counterparts (Appendix Fig S1J). These results further reinforce our hypothesis of epithelial-derived profibrotic signals capable of activating the IPF-MCs epigenetic imprint of their origin.

As MCs have been shown to shape epithelial homeostasis (Zepp et al, 2017; Cassandras et al, 2020), we next assessed whether activated MCs in turn influence AECs. To test this, we injured AECs for $3 \mathrm{~h}$ in co-culture with MCs, followed by substitution with either (i)

Figure 1. Injured epithelial secretions initiate a fibrotic cascade with mesenchymal cells.

A Schematic workflow of the co-culture experiment in which AECs were subjected to apical TGF $\beta 1$ stimulation for $72 \mathrm{~h}$ in the co-culture system. Mono-cultures were employed as negative controls.

B Representative E-cadherin (E-cad) and DAPI immunofluorescence images of AECs treated apically with TGF $\beta 1$ or vehicle control after $72 \mathrm{~h}$ in the co-culture system (scale bars $25 \mu \mathrm{m}$ ).

C ELISA shows decreased E-cad levels in TGF $\beta 1$-treated AECs after $72 \mathrm{~h}$ in the mono- and co-culture systems $\left(n=6\right.$ biological replicates, mean $+\mathrm{s.d}$., ${ }^{\star * *} P<0.001$, ANOVA/Tukey's).

D Representative $\alpha$ SMA and DAPI staining of healthy and IPF-derived MCs from the mono- and co-culture systems after $72 \mathrm{~h}$ apical treatment with TGF $\beta 1$. Note an increase in $\alpha$ SMA levels only in the presence of AECs and further enhanced in IPF-MCs (scale bars $100 \mu \mathrm{m}$ ).

E ELISA shows increased $\alpha$ SMA levels in a TGF $\beta 1$ dose-dependent manner from MCs and IPF-MCs in the co-culture system. Note a stronger $\alpha$ SMA response in IPFMCs (mean + s.d., $n=3$ biological replicates from 6 MCs and 6 IPF-MCs donors, ${ }^{\star \star} P=0.0014$, ${ }^{* \star \star *} P<0.0001$, nonlinear regression followed by ANOVA/Sidak's).

$\mathrm{F} \quad$ Schematic workflow of the substitution system in which AECS were subjected to TGF $\beta 1$ stimulation for $3 \mathrm{~h}$ in co-culture with MCs, after which (1) MCs or (2) AECs were replaced with untreated counterparts or (3) both fresh media and untreated MCs for a further $72 \mathrm{~h}$.

$\mathrm{G}, \mathrm{H} 72 \mathrm{~h}$ after substitutions in the co-culture, aSMA and E-cad levels of MCs and AECs, respectively, were measured with ELISA and normalised to the control. Note replacement of injured AECs with untreated AECs or replacement of media and MCs attenuates $\alpha$ SMA expression and increases E-cad levels (mean + s.d., ns $=$ non-significant, ${ }^{* *} P<0.001,{ }^{* *} P<0.01, n=3$ biological replicates with 6 MCs donors, repeated measurement ANOVA for MCs or ordinary ANOVA for AECs with Dunnett's post hoc test).

See also Appendix Fig S1. 
A

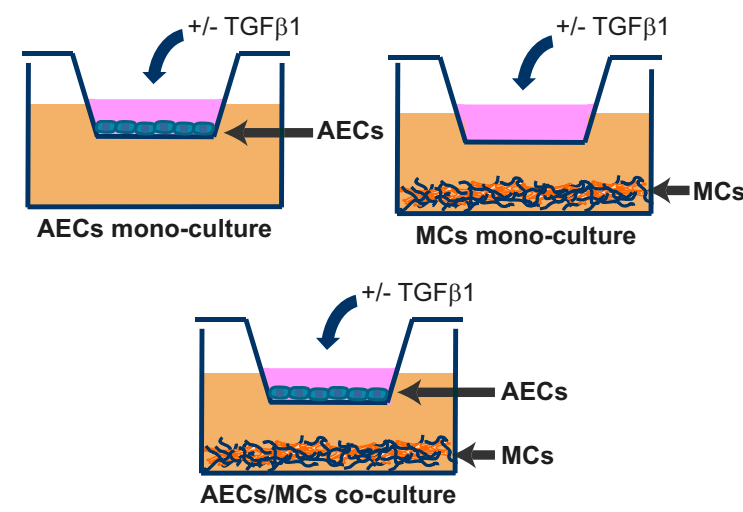

D

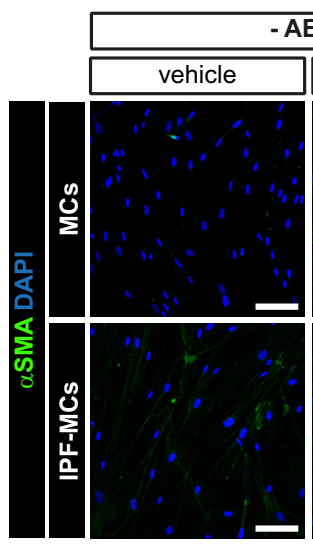

F

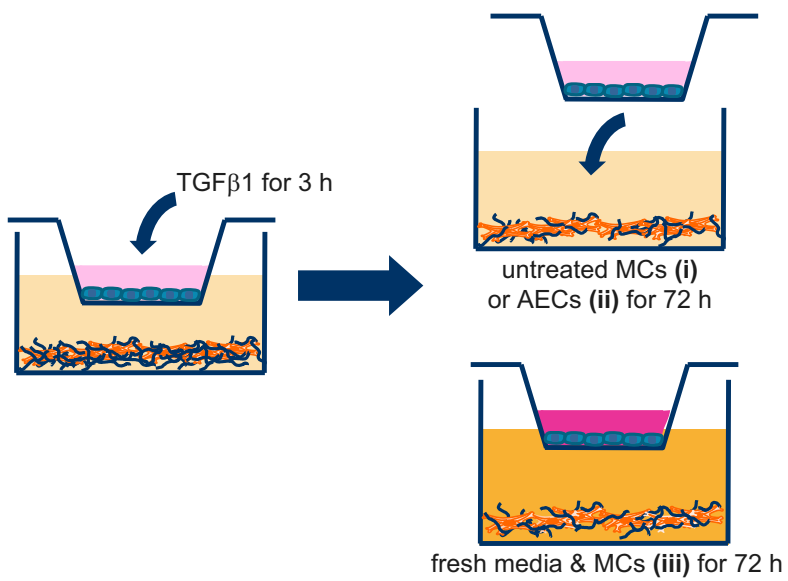

B

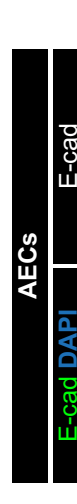

C

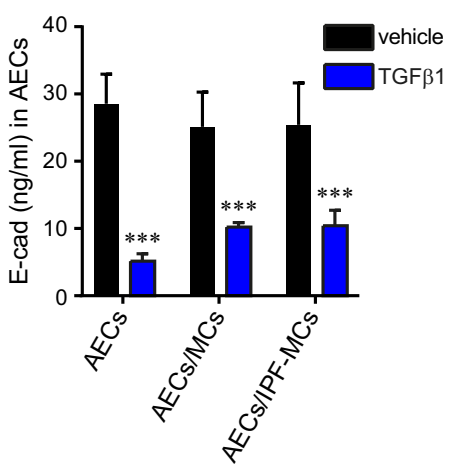

E

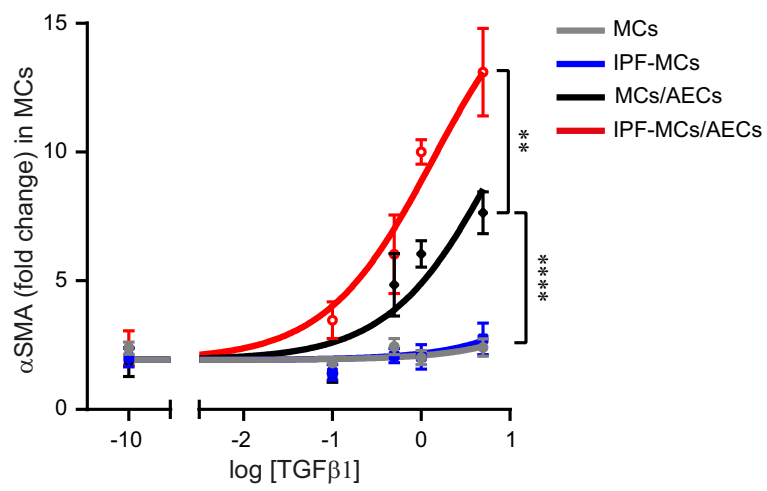

H

G

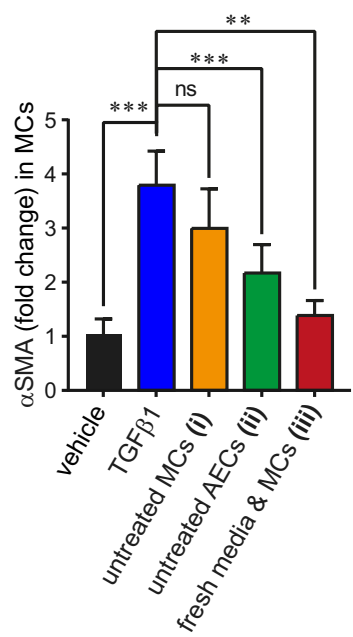

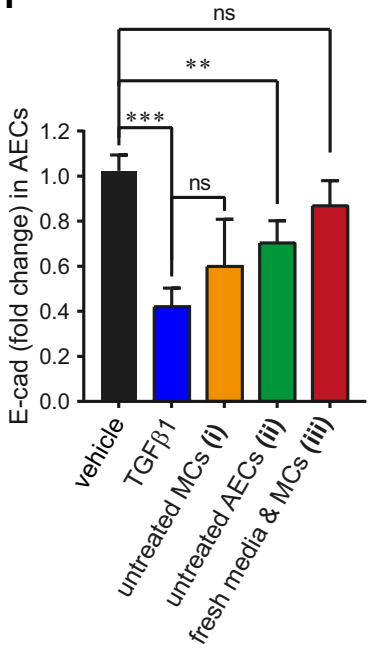

Figure 1.

untreated MCs, (ii) untreated AECs or (iii) fresh media and MCs for a further $72 \mathrm{~h}$ (Fig 1F). We observed an induction of $\alpha$ SMA levels in substituted MCs (i), consistent with injured epithelial-driven fibroblast-to-myofibroblast transition (FMT). Accordingly, replacement with untreated AECs (ii) or a combination of fresh media and MCs (iii) decreased aSMA expression in MCs (Fig 1G). Furthermore, the untreated AECs substituted into the MCs co-culture exhibited a significant decrease in E-cad levels (ii), indicating that activated MCs damage the epithelium in return (Fig $1 \mathrm{H}$ ). Collectively, these results demonstrate that injured AECs initiate the activation of MCs, which induces further epithelial damage, profibrotic mediator release and enhanced FMT.

Recently, it has become apparent that epithelial remodelling is a key driver of IPF; however, the underlying mechanisms remain unclear (Boesch et al, 2020; Habermann et al, 2020; Selman \& Pardo, 2020). Therefore, we focused on unveiling the pathological drivers of aberrant lung epithelial remodelling and its fibrotic consequences. 


\section{Polycomb mediates fibrotic epithelial secretions in response to IPF-related injury}

We next sought to identify the molecular programmes that are involved in the epithelial-driven fibrotic crosstalk. After $72 \mathrm{~h}$ of coculture following epithelial injury, transcriptional and secretomic profiles of the AECs and MCs were analysed using RNA sequencing (RNA-seq) and mass spectrometry, respectively. We found 1,732 significantly differentially expressed genes (DEGs) in AECs upon damage, whereas 3,778 genes were deregulated in MCs $(q<0.05$; Fig 2A, Appendix Fig S2A and Dataset EV1). Furthermore, we detected 60 significantly differentially secreted proteins in response to epithelial injury ( $q<0.05$; Fig 2B and Dataset EV2), which showed a positive correlation with RNA-seq data (Appendix Fig S2B and C). Intriguingly, gene ontology (GO) analysis revealed enriched genes/ proteins involved in the development and progression of fibrotic disease (marked by asterisk in Fig 2A), including regulators of mRNA metabolic processes, endoplasmic reticulum stress, unfolded protein response and ECM deposition (Fig 2C-E and Dataset EV3). Furthermore, gene set enrichment analysis (GSEA) of DEGs from each compartment independently mapped to an IPF transcriptomic database (Kusko et al, 2016; Habermann et al, 2020) revealed a significant correlation with an IPF whole lung signature as well as certain IPF epithelial and mesenchymal subpopulations from a single-cell atlas (Fig 2F and Table EV1A-C). A major finding from these analyses was that the injured AECs in our co-culture system displayed an IPF transitional alveolar type 2 epithelial cells signature (Fig 2F), an epithelial subgroup implicated in aberrant repair programmes in the IPF lung, leading to defective alveolar function and thus fibrotic progression (Habermann et al, 2020). These findings collectively corroborate the relevance of our in vitro model to IPF.

To gain insights into the gene regulatory pathways underlying the observed fibrotic phenotype in our model, we performed an unbiased GSEA approach using molecular signatures of genetic, chemical perturbations and oncogenic database (Subramanian et al, 2005). The results showed that genes marked with H3K27me3 (Meissner et al, 2008), as well as known targets of the polycomb subunits EZH2 (Bracken et al, 2006) and SUZ12 (Pasini et al, 2007), were significantly represented among the top-ranked gene sets (Fig 2G and Table EV2A-C). This was intriguing, as multiple genes linked to DNA methylation in injured AECs (marked in blue,
Fig 2A) were significantly downregulated and enriched in the GO term of transcriptional regulation (Fig 2D). Collectively, these observations suggest that injured AECs activate the fibrotic response through altered polycomb activity. Importantly, polycomb target genes were also significantly enriched in the set of deregulated genes from injured AECs in the mono-culture (Appendix Fig S2D and Table EV2D), indicating that the polycomb-mediated fibrotic response in AECs is a direct effect of TGF $\beta 1$-induced injury.

To test this hypothesis, we measured global levels of EZH2 and its functional marker $\mathrm{H} 3 \mathrm{~K} 27 \mathrm{me}$. Interestingly, epithelial damage reduced $\mathrm{H} 3 \mathrm{~K} 27 \mathrm{me} 3$ levels, whereas total EZH2 levels increased (Fig 2H and Appendix Fig S2E). We next determined H3K27me3 and EZH2 occupancy on profibrotic genes detected in our RNA-seq data by chromatin immunoprecipitation (ChIP). Accordingly, we observed a decrease in $\mathrm{H} 3 \mathrm{~K} 27 \mathrm{me} 3$ occupancy at promoters of profibrotic genes in response to TGF $\beta 1$-induced injury; however, EZH2 occupancy remained unchanged (Appendix Fig S2F and G).

\section{Injured AECs triggers an EZ switch from EZH2-PRC2 to EZH1-PRC2}

The desynchronised changes in gene occupancy of H3K27me3 and EZH2 prompted us to examine non-canonical functions of EZH2. Phosphorylation of EZH2 on T311 (ph-EZH2) has been shown to disrupt the interaction between EZH2 and SUZ12 (Wan et al, 2018). Concurrently, chronic TGF $\beta 1$ treatment increased nuclear ph-EZH2 levels on T311 (Fig 3A and Appendix Fig S3A), suggesting a PRC2independent, non-canonical role of EZH2 in injured AECs. We therefore hypothesised that TGF $\beta 1$-induced injury leads to disruption of the EZH2-SUZ12 interaction, followed by a small reduction in global H3K27me3 levels. The EZH2 homolog, EZH1, has been previously shown to compensate for the loss of EZH2 in the PRC2 complex (Aoyama et al, 2018; Lavarone et al, 2019); we thus sought to ascertain whether an EZ switch from EZH2-PRC2 to EZH1-PRC2 occurs, to sustain PRC2 function. Co-immunoprecipitation (co-IP) experiments uncovered such an interaction between EZH1 and SUZ12 in which chronic TGF $\beta 1$ treatment enhanced SUZ12-bound EZH1, while simultaneously reducing SUZ12-bound EZH2 (Fig 3B). To validate this EZ switch at the single-gene level, we investigated EZH1, ph-EZH2 (T311) and H3K27me3 occupancy on profibrotic genes and other known PRC2 targets. ChIP analysis confirmed an

Figure 2. Polycomb mediates the fibrotic response in the co-culture system.

A Hierarchical clustering of differentially regulated transcripts from RNA-seq between vehicle and TCF $\beta 1$-treated AECs in the co-culture $(P$-adj $<0.05$, $t$-test with Benjamini-Hochberg Correction). Significantly regulated profibrotic genes (marked by asterisk) and histone/DNA methyltransferase encoded genes (marked in blue) are listed on the right side.

B Volcano plot representing logarithmic ratio of differentially secreted proteins from proteomics analysis of co-culture medium upon apical TGF $\beta 1$ stimulation $(P$-adj $<0.05, t$-test), with examples of profibrotic secreted proteins (red dots indicating significantly upregulated proteins, blue dots indicating significantly downregulated proteins, and grey dots indicating no significant change in protein expression levels).

C-E Gene Ontology (GO) analysis of differentially expressed genes/proteins ( $P$-adj $<0.05$, hypergeometric test) that are enriched in (C) TGF $\beta 1$-treated, (D) vehicletreated $A E C S$ and $(E)$ differentially secreted proteins.

F Gene set enrichment analysis (GSEA) shows enrichment of an IPF transcriptional and cellular phenotype in TGF $\beta 1$-injured AECs/MCs co-culture system (Kolmogorov-Smirnov test). Note, injured AECs displays an IPF transitional alveolar type 2 cells signature.

G GSEA shows enrichment of genes defined as polycomb targets in injured AECs/MCs co-culture (Kolmogorov-Smirnov test with Benjamini-Hochberg correction).

$\mathrm{H} \quad$ Representative H3K27me3 and EZH2 immunofluorescence images and box plots (minimum, first quartile, median, third quartile and maximum) showing decreased H3K27me3 levels but increased total EZH2 levels in TGF $\beta 1$-injured AECs in co-culture with MCs ( $n=5$ biological replicates with $>50$ cells per experiment, scale bars $50 \mu \mathrm{m},{ }^{\star} P<0.05$, unpaired $t$-test).

See also Appendix Fig S2. 
A

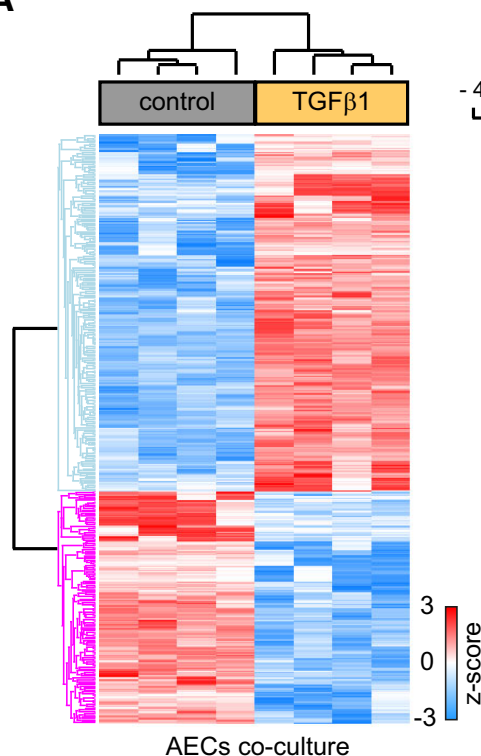

B

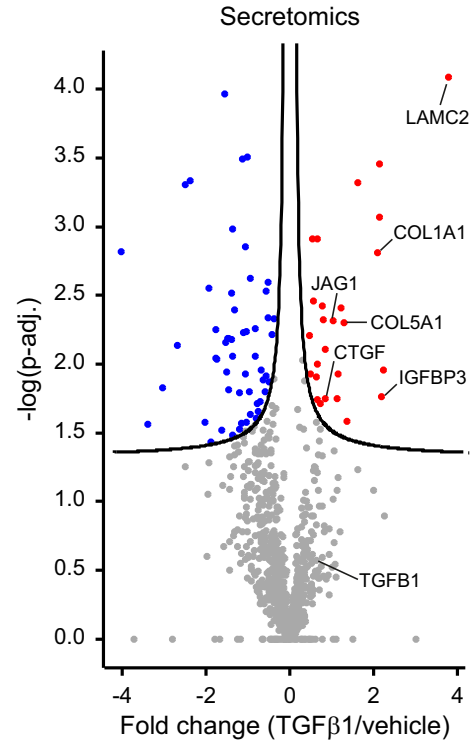

$\log _{2}$ fold change
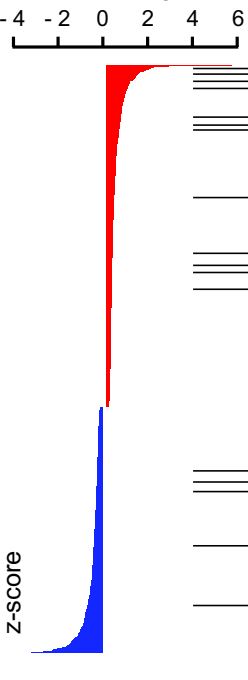

\section{6}

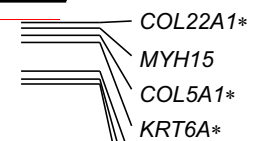

$\longrightarrow$ TGFB1*

$\Longrightarrow$ CEACAM5*

$\longrightarrow$ ATF3*

TP63*

MUC1*

$\mathrm{CDH} 2 *$

PLAUR*

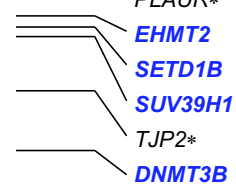

E
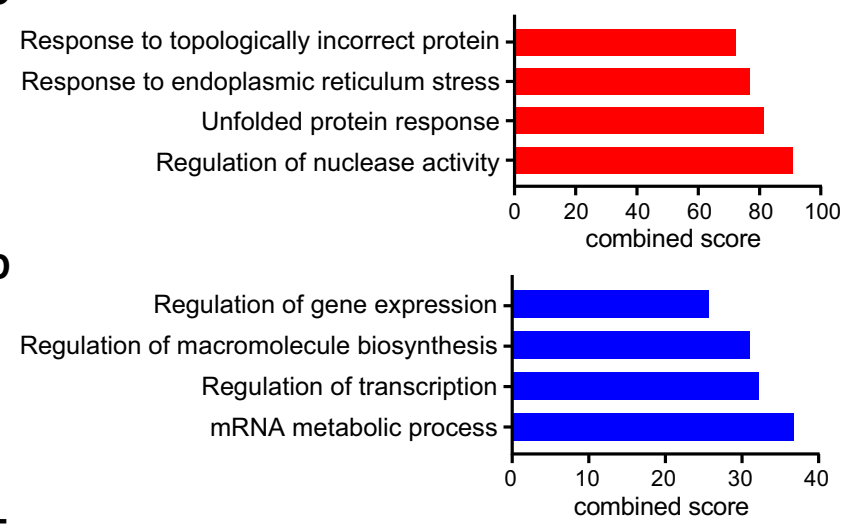

Protein complex subunit organization -

Extracellular matrix organization

Collagen fibril organization

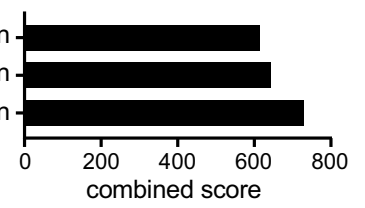

F

IPF transitional AEC2

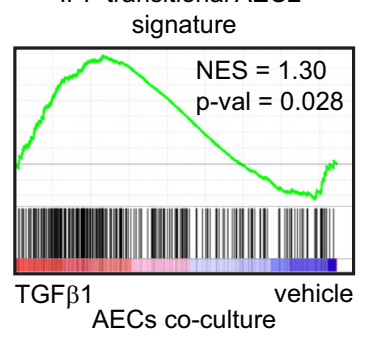

IPF myofibroblasts signature
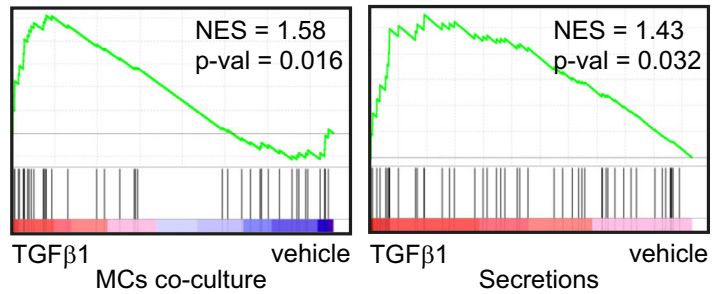

G

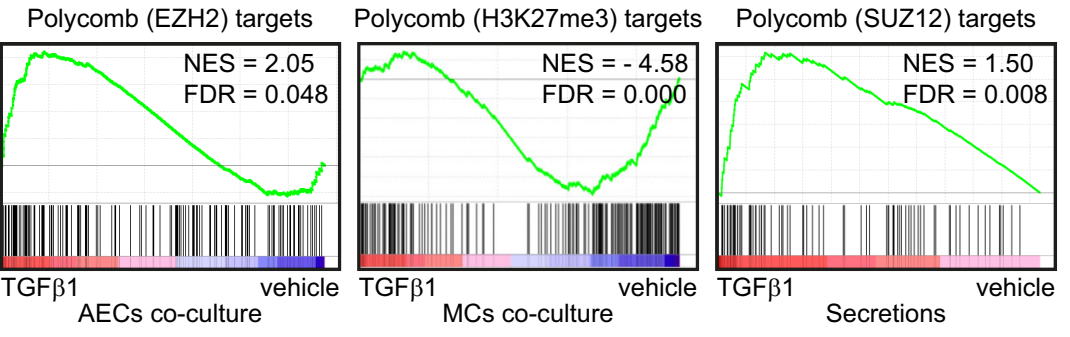

H

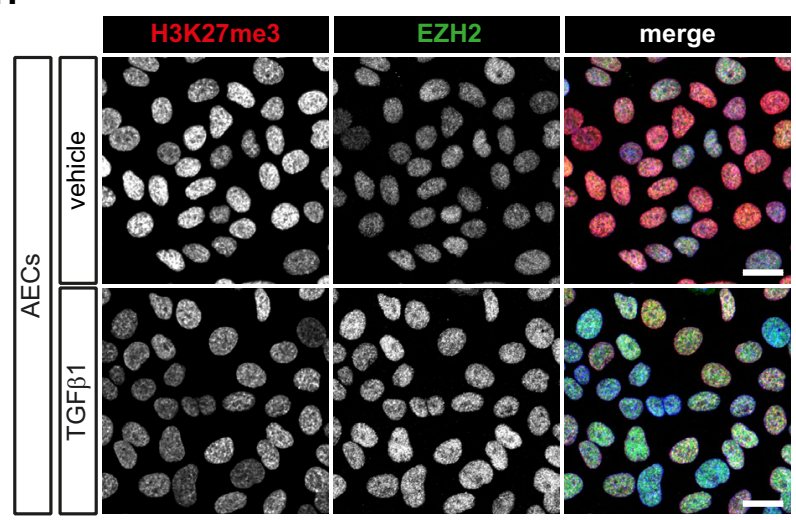

H3K27me3
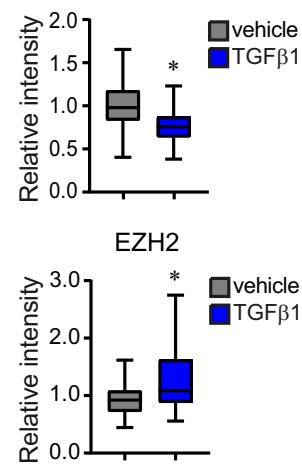

Figure 2. 
enrichment of ph-EZH2 occupancy at gene bodies of profibrotic genes in response to injury (Fig 3C), but not at non-target genes (Appendix Fig S3B). Furthermore, EZH1 occupancy was increased at promoters of non-fibrotic genes to maintain H3K27me3 levels (Fig 3D and E), consistent with previous reports of EZH1 compensation for EZH2 deficiency (Aoyama et al, 2018; Lavarone et al, 2019).

\section{Non-canonical EZH2 promotes a profibrotic response in injured AECs}

We next analysed the contribution of ph-EZH2 in fine-tuning profibrotic genes. As expected, we observed an attenuation of ph-EZH2 upon RNAi-mediated depletion of EZH2 in injured AECs (Appendix Fig S3C). Subsequently, EZH2-depleted AECs led to a less pronounced upregulation of profibrotic genes in AECs in response to injury (Appendix Fig S3D). This in turn prevented MCs from undergoing FMT in the co-culture system (Appendix Fig S3E).

To specifically dissect a functional relationship between ph-EZH2 and fibrosis, we expressed either wildtype (WT) EZH2, phosphomimetic EZH2-T311D or phosphorylated-deficient EZH2-T311A in EZH2-deleted AECs. As expected and in line with a previous study (Wan et al, 2018), the phosphomimetic T311D showed a significant reduction of H3K27me3 levels compared to WT or T311A (Fig 3F). Importantly, in contrast to $\mathrm{T} 311 \mathrm{~A}$, addition of $\mathrm{T} 311 \mathrm{D}$ promoted the expression of profibrotic genes (Fig $3 G$ and Appendix Fig S3F). Collectively, these data provide a direct evidence for our hypothesis that injured epithelium activates the fibrotic cascade in a noncanonical EZH2-dependent manner.

\section{Nuclear TAK1 regulates phosphorylation of EZH2}

To determine the upstream kinase that mediates EZH2 phosphorylation, we performed functional kinase enrichment analysis (KEA) of DEGs from AECs and identified TAK1 (encoded by MAP3K7) as a top kinase candidate (Fig 4A and Dataset EV4). We next assessed whether the fibrotic response in injured AECs depends on TAK1 activity. Cytoplasmic and nuclear fractions analysis showed increased levels of active TAK1 (ph-TAK1) in the cytoplasm $24 \mathrm{~h}$ post-injury (Appendix Fig S4A). Interestingly, nuclear translocation of ph-TAK1 was detected at $48 \mathrm{~h}$ and coincided with increased nuclear ph-EZH2 levels (Fig 4B). Co-IP analysis unveiled a direct interaction between ph-TAK1 and ph-EZH2 in injured AECs (Fig 4C), indicating that activated TAK1 might phosphorylate EZH2 in the fibrotic lung.

We next evaluated whether abrogation of TAK1 function would be sufficient to impair ph-EZH2 levels. To explore this, we administered the TAK1 inhibitor 5Z-7-Oxozeaenol (5-OZ) (Iriondo et al, 2018) to the AECs/MCs co-culture model that underwent TGF $\beta 1$-induced injury. As expected, 5-OZ hindered TAK1 activity and resulted in attenuated ph-EZH2 levels in injured AECs (Fig 4D). Moreover, TAK1 inhibition reduced POL2 occupancy on profibrotic genes (Fig 4E), thus preventing the fibrotic effect on MCs (Appendix Fig S4B). Accordingly, RNAi-mediated depletion of MAP3K7 (encoding for TAK1) in AECs prevented ph-EZH2 accumulation and subsequently silenced the fibrotic crosstalk with MCs (Appendix Fig S4 C and D).

Finally, to assess whether TAK1 can directly phosphorylate EZH2, we performed in vitro kinase assays and found that TAK1 phosphorylates EZH2 on T311 (Appendix Fig S4E). While 5' adenosine monophosphate-activated protein kinase $\alpha$ (AMPK $\alpha$ ) has been previously shown to be the responsible kinase for this phosphosite (Wan et al, 2018), AMPK $\alpha$ levels as well as EZH2-bound active AMPK $\alpha$ (ph-AMPK $\alpha$ ) were unaltered in injured AECs compared to control (Appendix Fig S4F and G), indicating that AMPK $\alpha$ is not the main kinase responsible for EZH2 phosphorylation in our fibrotic model. Collectively, these data demonstrate that the kinase activity of nuclear TAK1 is required to phosphorylate EZH2, thus facilitates its PRC2-independent function in the fibrotic lung.

\section{A novel transcriptional complex including EZH2, POL2 and nuclear actin fine-tunes profibrotic gene expression}

To unravel the mechanisms of the ph-EZH2-mediated profibrotic gene expression, we investigated the potential binding partners of EZH2 in injured AECs. It has been shown that actin cytoskeleton remodelling is an important hallmark of fibrosis (Rana et al, 2018). Actin is constantly shuttled between the cytoplasm and the nucleus, where it has been shown to associate with chromatin remodelling complexes and POL2 to facilitate transcriptional activity (Le et al,

Figure 3. TGFß1-induced injury triggers an EZ switch from EZH2-SUZ12 to EZH1-SUZ12 via EZH2-T311 phosphorylation in AECs.

A Representative simple western analysis (Peggy Sue) of ph-EZH2 and quantification shows increased ph-EZH2 levels on T311 in AECs subjected to apical TGF $\beta 1$ for $72 \mathrm{~h}$ compared to vehicle treatment (mean + s.d., $n=5$ biological replicates, ${ }^{\star \star \star} P=0.0008$, unpaired $t$-test).

B Representative simple western analysis (Peggy Sue) of SUZ12 immunoprecipitates shows co-precipitation of EZH1 and EZH2 in AECs. TGF $\beta 1$-induced injury leads to the EZ switch from SUZ12-bound EZH2 to EZH1. Unspecific IgG binding was used as a negative control. A representative from 3 biological replicates is shown.

C ChIP-qPCR shows increased ph-EZH2 occupancy at gene bodies of profibrotic genes in AECs subjected to TGF $\beta 1$ for 72 h. Note no changes in ph-EZH2 levels at nontarget genes (mean + s.d., $n=3$ biological replicates). Unspecific IgC was used as negative control. ChIP-qPCR for non-fibrotic genes is shown in Appendix Fig S3A.

D ChIP-qPCR shows increased EZH1 occupancy at promoters of non-fibrotic genes in AECs subjected to apical TGF $\beta 1$ for $72 \mathrm{~h}$ (mean + s.d., $n=3$ biological replicates). Unspecific IgG was used as negative control.

E ChIP-qPCR shows no changes in H3K27me3 at promoters of non-fibrotic genes in AECs subjected to apical TGF $\beta 1$ for $72 \mathrm{~h}$ (mean + s.d., $n=3$ biological replicates). Unspecific IgG was used as negative control.

F Representative simple western analysis (Peggy Sue) and quantifications (right panels) for EZH2 and H3K27me levels from EZH2-deleted AECs (sgEZH2) which were reintroduced empty vector (EV), T311 wildtype (WT), a phosphorylated-deficient T311A or a phosphomimetic T311D form of EZH2. Quantifications show mean + s.d. ( $n=4$ biological replicates, ${ }^{*} P<0.05,{ }^{* *} p<0.01$, Kruskal-Wallis/Dunn's).

G EZH2-deleted AECs (sgEZH2) reintroducing empty vector (EV), T311 wildtype (WT), a phosphorylated-deficient T311A or a phosphomimetic T311D form of EZH2 were quantified for the expression of profibrotic genes. Vehicle and TGF $\beta 1$-treated AECs (sgNEG + vehicle/TGF $\beta 1$ ) were used as control. mRNA levels are normalised to HPRT1 expression. (mean + s.d., $n=4$ biological replicates, ${ }^{*} p<0.05$, ${ }^{\star *} p<0.01$, ${ }^{\star * *} P<0.001$, ${ }^{* * *} p<0.0001$, Kruskal-Wallis/Dunn's).

See also Appendix Fig S3.

Source data are available online for this figure. 
A

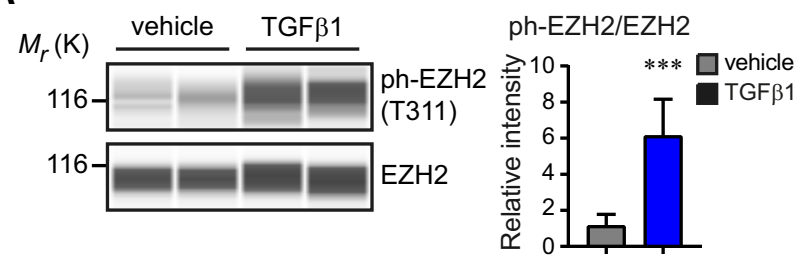

B

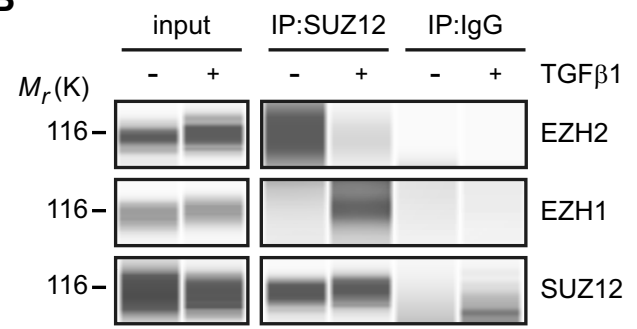

C

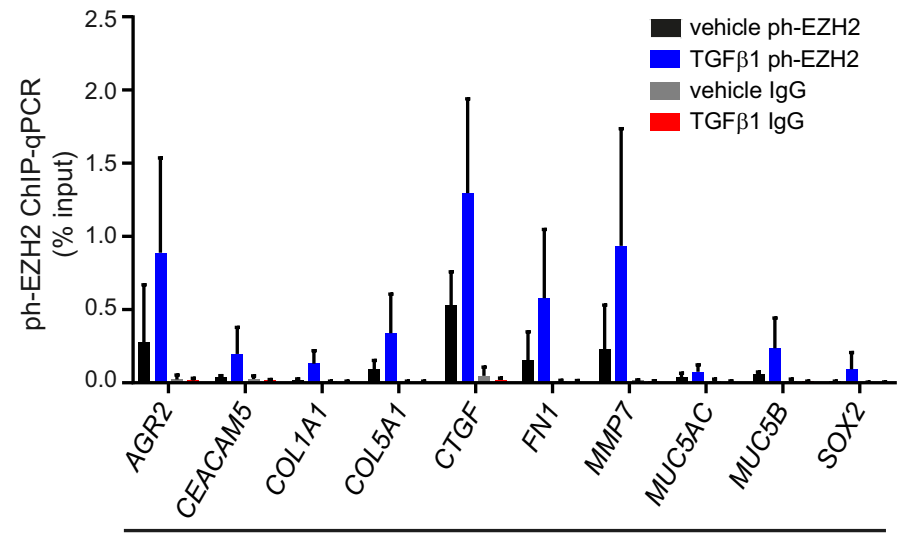

Profibrotic genes
D

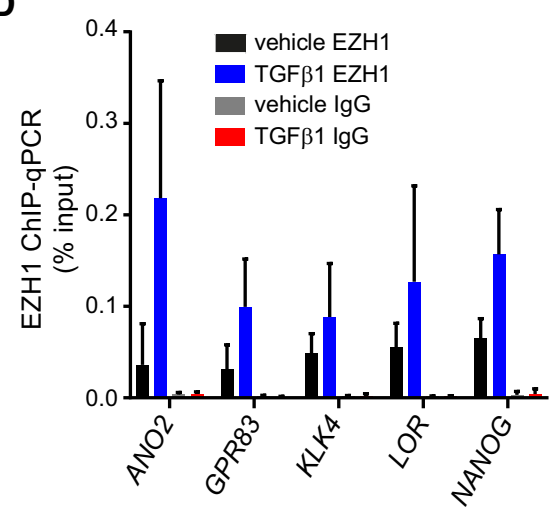

E

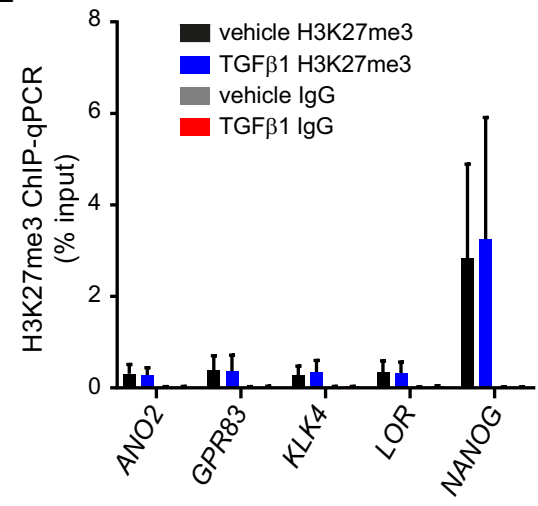

$\mathbf{F}$
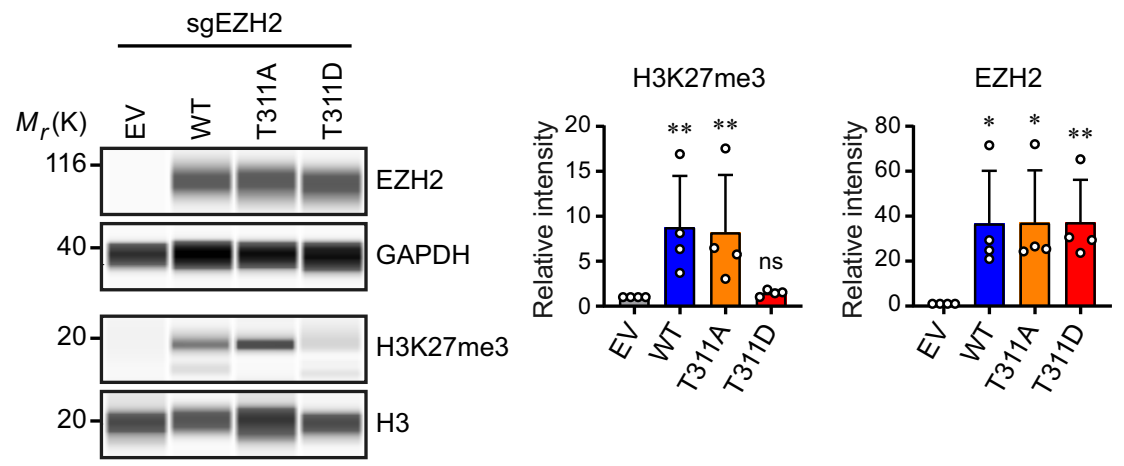

G

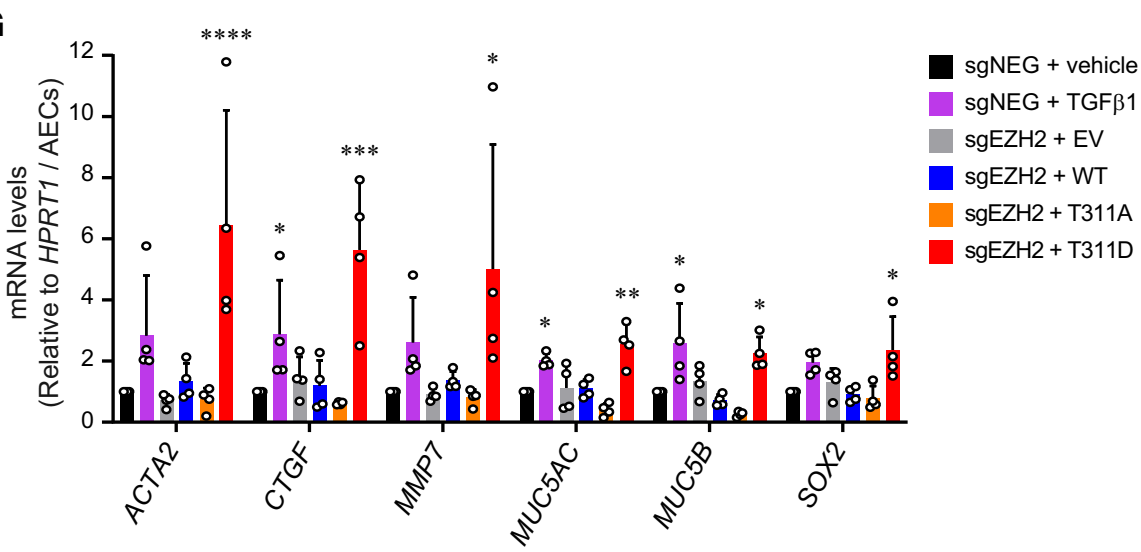

Figure 3.

2016; Wei et al, 2020). Since transcriptional regulation was among the most enriched GO term in injured AECs (Fig 2D), we hypothesised that ph-EZH2 mediates the transcriptional process of the profibrotic genes, specifically through POL2 and nuclear actin. As anticipated, nuclear fractionation confirmed that injured AECs increased levels of nuclear actin after $48 \mathrm{~h}$ of stimulation (Fig 5A). Intriguingly, analysis of the EZH2 co-IP revealed an interaction between ph-EZH2, POL2 and actin in injured AECs (Fig 5B). We further validated this novel interaction by performing reversed co-IP for the active form of POL2 (marked by its phosphorylation on serine 2; S2p; Suh et al, 2016) and observed that TGF $\beta 1$-induced injury promoted the formation of a novel fibrotic transcriptional complex consisting of ph-EZH2, POL2-S2p and nuclear actin (Appendix Fig S5A).

The emergence of an interaction between ph-EZH2 and POL2 led us to focus on the existence of poised and elongated forms of POL2 (Suh et al, 2016) at profibrotic genes. Analysis of the occupancy of poised POL2 (phosphorylated on S5) and elongated S2p revealed an increase in POL2-S5p occupancy within the first $24 \mathrm{~h}$ upon injury, whereas POL2-S2p occupancy only became visible after $48 \mathrm{~h}$ (Fig 5C-F and Appendix Fig S5B and C), the time when increased nuclear actin levels were detected. Furthermore, inhibition of POL2-S2p by DRB (5,6-dichlorobenzimidazole 1- $\beta$-D-ribofuranoside; Fraser et al, 1978) did not disrupt the 
A

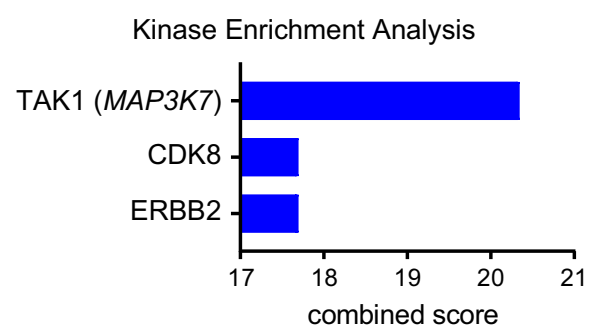

B
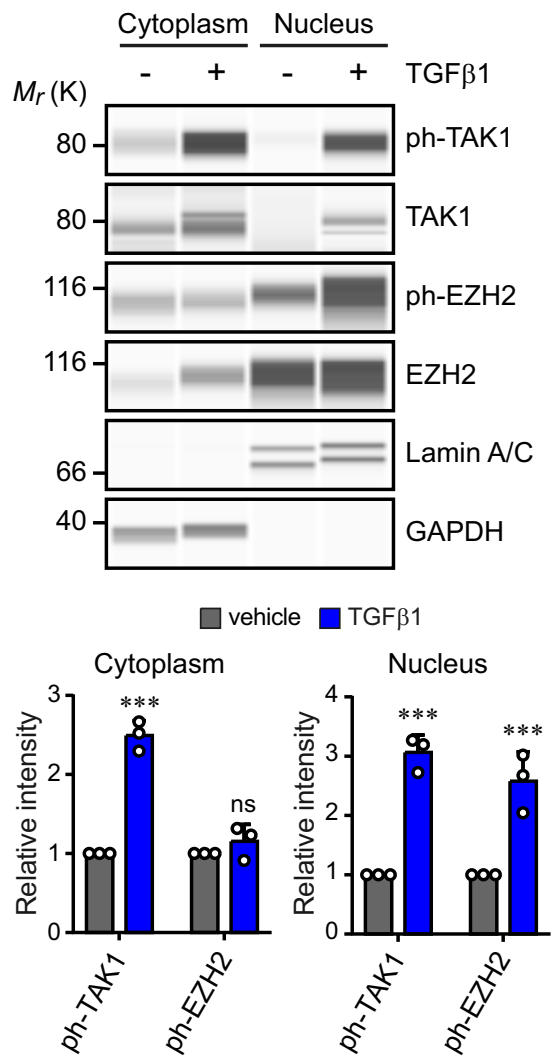

C

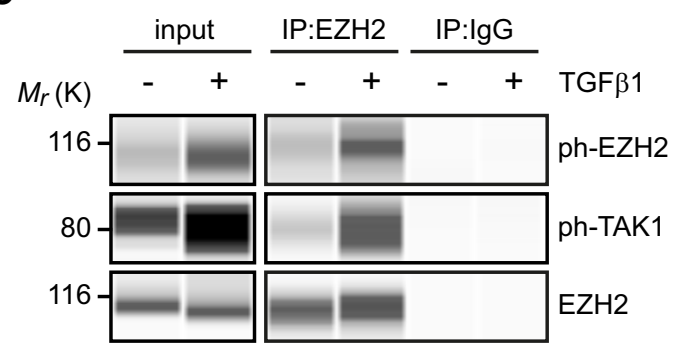

D

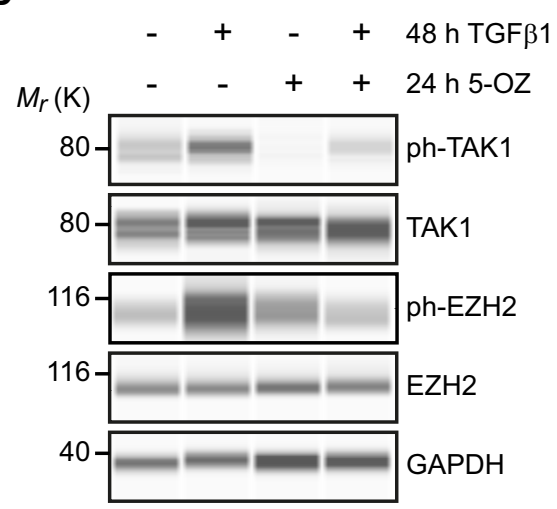

E

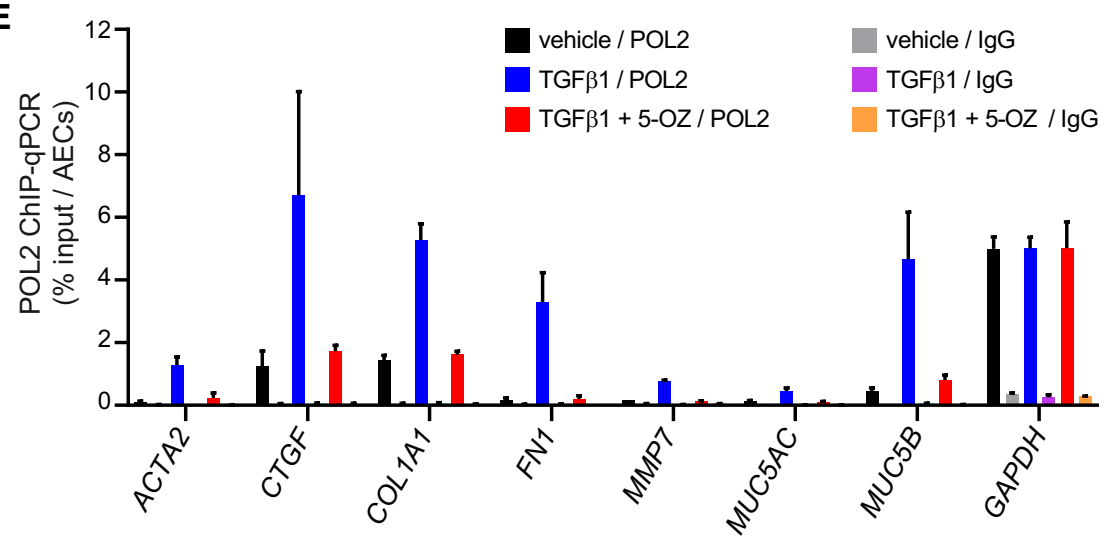

Figure 4. Nuclear TAK1 mediates ph-EZH2 in injured AECs to control profibrotic response.

A Kinase enrichment analysis showing enrichment of TAK1 (encoded by MAP3K7) in injured AECs ( -adj < 0.05, hypergeometric test with Benjamini-Hochberg correction).

B Nuclear fractionation followed by simple western analysis (Peggy Sue) of AECs exposed to $72 \mathrm{~h}$ of TGF $\beta 1$ in the co-culture system shows an increase in phosphorylated TAK1 and a parallel increase in ph-EZH2. Quantifications (lower panels) show mean + s.d., $n=3$ biological replicates (ns $=$ non-significant, $\star * * P<0.001$, Kruskal-Wallis/Dunn's).

C Simple western analysis (Peggy Sue) of EZH2 immunoprecipitates shows increased co-precipitation of ph-EZH2 (T311) and ph-TAK1 in injured AECs. Unspecific IgG was used as negative control. A representative from three experiments is shown.

D Simple western analysis (Peggy Sue) shows a TAK1-dependent enrichment of ph-EZH2 levels in injured AECs. Note: TAK1 inhibitor (5-OZ) attenuates increased ph-EZH2 levels in injured AECs. Quantifications (right panels) show mean + s.d., $n=5$ biological replicates (ns $=$ non-significant, ${ }^{* \star} P=0.0026,{ }^{* \star *} p<0.001$, ANOVA/Tukey's).

E ChIP-qPCR shows diminished POL2 occupancy on profibrotic genes in injured AECs subjected to $5-0 Z$ (mean + s.d., $n=3$ biological replicates). Unspecific IgG was used as negative control.

See also Appendix Fig S4

Source data are available online for this figure.

interaction between ph-EZH2 and POL2 (Appendix Fig S5D), suggesting that S2 phosphorylation of POL2 is not required for its binding with ph-EZH2. Together, these data indicate that TGF $\beta 1$-induced injury activates profibrotic gene expression in a stepwise process: (i) acute TGF $\beta 1$ signalling within the first $24 \mathrm{~h}$ induces a poised state of profibrotic genes, which is independent of the TAK1-EZH2 axis; and (ii) such a poised state ensures only chronic and sustained injury are capable of activating transcription through TAK1-mediated liberation of EZH2 from PRC2 after $48 \mathrm{~h}$. 
A
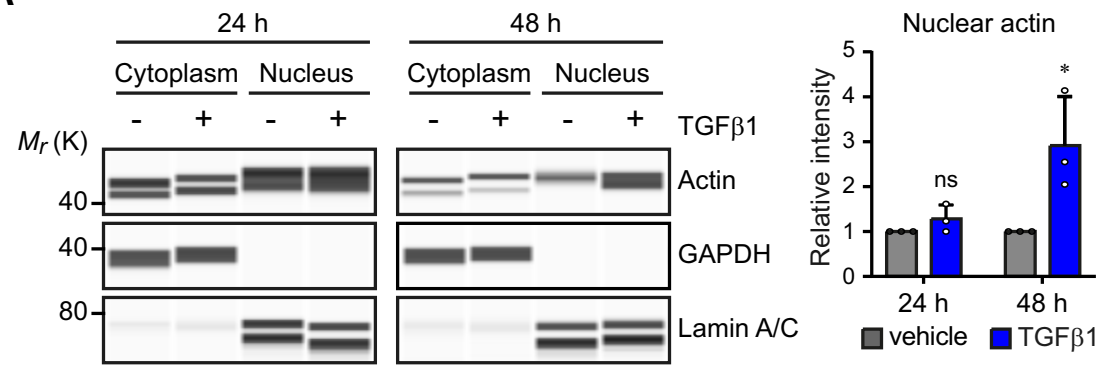

C

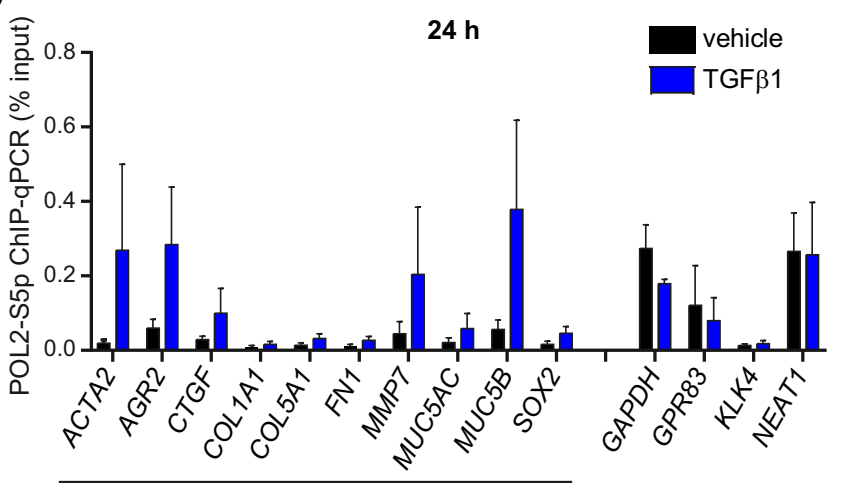

Profibrotic genes

E

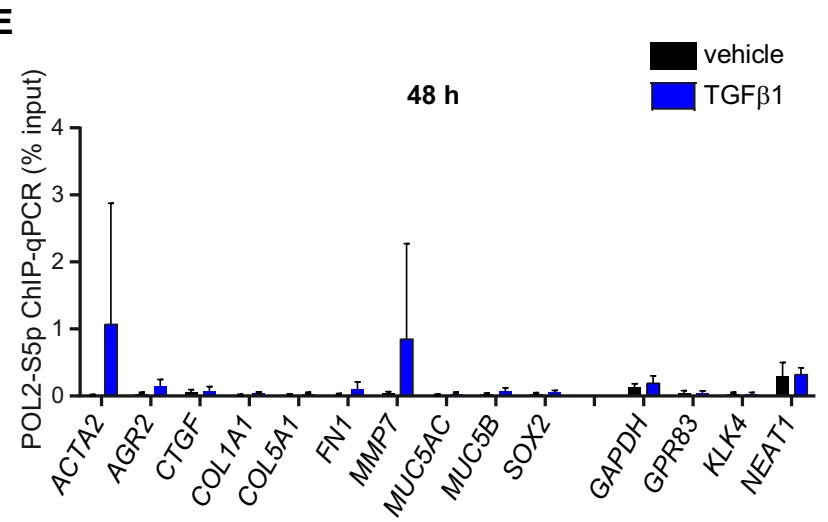

Profibrotic genes
D

B

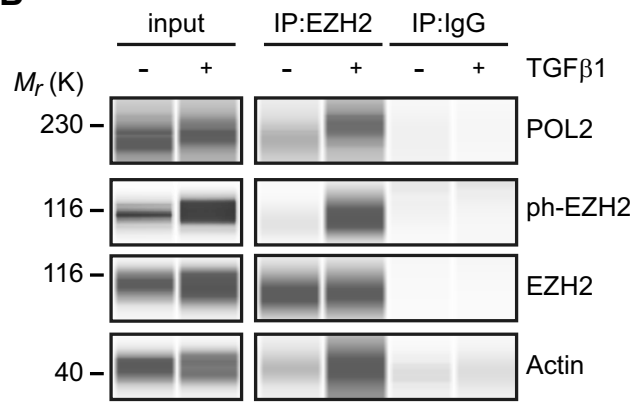

$24 \mathrm{~h}$

vehicle

TGF $\beta 1$

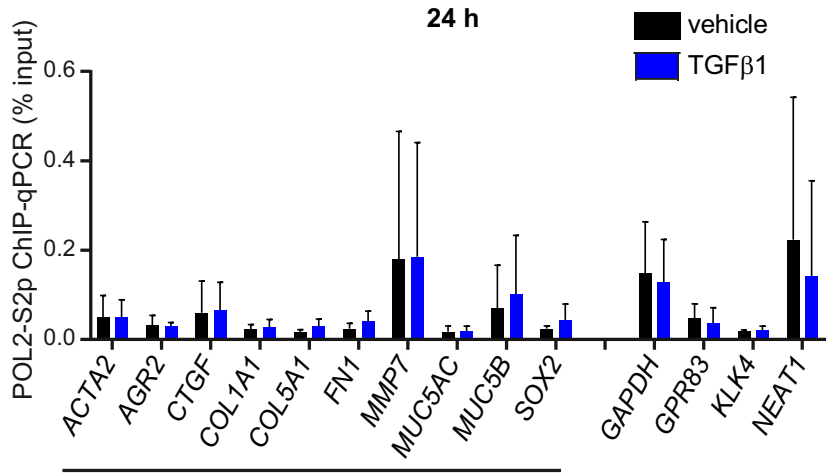

Profibrotic genes

$\mathbf{F}$

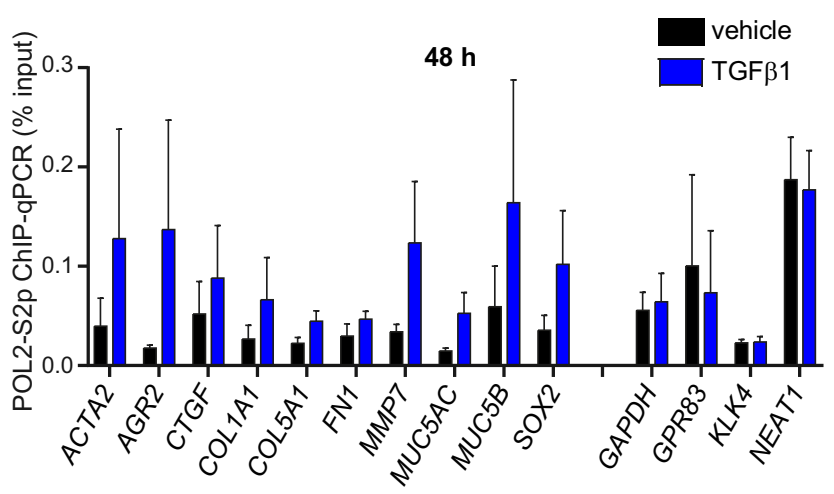

Profibrotic genes

Figure 5. TGFß1 mediates profibrotic response through a novel transcriptional complex of EZH2, POL2 and nuclear actin.

A Nuclear fractionation followed by simple western analysis (Peggy Sue) shows an increase in nuclear actin in AECs exposed to TGF $\beta 1$ for $48 \mathrm{~h}$. Quantification (right panel) shows mean + s.d., $n=3$ biological replicates, $\mathrm{ns}=$ non-significant, ${ }^{*} P=0.014$, ANOVA/Tukey's).

B Simple western analysis (Peggy Sue) of EZH2 co-immunoprecipitates shows increased levels of EZH2-bound POL2, ph-EHZ2 and actin in injured AECs. Unspecific IgG binding was used as a negative control. A representative from 3 biological replicates is shown.

C, D ChIP-qPCR shows increased occupancy of (C) POL2-S5p at promoters of profibrotic genes in AECs subjected to TCF $\beta 1$ for 24 h, whereas no enrichment of (D) POL2S2p at the gene bodies of these genes was detected. Negative IgG control is shown in Appendix Fig S5B (mean + s.d., $n=3$ biological replicates).

E, F ChIP-qPCR shows increased occupancy of (E) POL2-S5p at promoters and (F) POL2-S2p at the gene bodies of profibrotic genes in AECs subjected to TGF $\beta 1$ for $48 \mathrm{~h}$. Negative IgG control is shown in Appendix Fig S5C (mean + s.d., $n=3$ biological replicates).

See also Appendix Fig S5.

Source data are available online for this figure.

\section{EZH2 catalytic activity is essential for the fibrotic transcriptional complex's activity}

As reported previously (Xu et al, 2012), EZH2 requires its methyltransferase activity when transitioning from a transcriptional repressor to a coactivator state. To test this, we used an EZH2 selective inhibitor GSK126 to block its catalytic activity (McCabe et al, 2012). As expected, GSK126 significantly reduced H3K27me3 levels in control AECs, but not in injured AECs (Fig EV1A), providing further evidence for an EZ switch in injured epithelial cells. Notably, inhibition of EZH2 catalytic activity decreased ph-EZH2 levels in injured cells (Fig EV1A). 
We next investigated the potential catalytic substrate of EZH2 In mammalian cells, methylation of a non-consensus lysine 7 of POL2 (POL2-K7m) has been shown to associate with early transcription and promoters of active genes (Dias et al, 2015; Voss et al, 2015). Interestingly, we detected a global increase in POL2-K7m levels and an interaction between this modification and non-PRC2 EZH2 in injured AECs (Fig EV1B and C). Strikingly, this enrichment was offset by inhibition of EZH2 activity (Fig EV1B). The loss of EZH2 activity was functionally relevant as it resulted in an attenuation of the fibrotic cascade (Fig EV1D). Together, these data show that EZH2 methyltransferase activity is required for its non-PRC2 function in the fibrotic transcriptional complex.

\section{Actin dynamics mediate POL2 activity within the fibrotic transcriptional complex}

Next, we sought to elucidate the role of actin in the fibrotic transcriptional complex. In line with previous data (Rana et al, 2018), we observed increased actin polymerisation in injured AECs. Interestingly, TGF $\beta 1$-induced actomyosin remodelling in injured AECs was attenuated by the Rho-kinase inhibitor Y27632 (Okuda et al, 2018) but not EZH2 depletion (Fig 6A). Moreover, blocking actomyosin remodelling disrupted the formation of the injury-induced fibrotic transcriptional complex (Fig 6B) and subsequently repressed the fibrotic crosstalk with MCs (Appendix Fig S6A-C). These experiments indicate that biomechanical signalling plays an important role in epithelial response to injury and subsequent nuclear signalling is mediated through actomyosin remodelling.

Since injured AECs mediate not only cytosolic F-actin remodelling but also enrichment of nuclear localised actin, we sought to investigate the functional consequences of nuclear actin in the fibrotic complex. Importin 9 (IPO9) has been shown to actively transport G-actin into the nucleus (Dopie et al, 2012); we therefore perturbed nuclear actin influx via RNAi-mediated depletion of IPO9 in AECs (Fig 6C). Intriguingly, IPO9 depletion resulted in decreased POL2-S2p levels, but showed no significant effect on ph-EZH2 in injured AECs (Fig 6D). Concomitantly, blocking nuclear actin influx attenuated profibrotic gene expression (Fig 6E). Together, these data demonstrate that nuclear actin is required for productive POL2 elongation, acting as a transcriptional modulator in response to TGF $\beta 1$-induced fibrosis.

\section{EZH2 promotes metaplastic differentiation of alveolar epithelium in vivo}

Finally, we investigated whether the EZH2-dependent transcriptional complex also directs the fibrotic response in vivo following injury. As the fibrotic cascade was triggered by epithelial EZH2, we predicted that lung epithelial cells lacking EZH2 activity would attenuate fibrotic remodelling in response to injury. To test this hypothesis, we used a lung fibrosis mouse model based on adenoassociated virus 6.2 (AAV)-mediated overexpression of TGF $\beta 1$, as described previously (Strobel et al, 2015). We first confirmed that the AAV-TGF $\beta 1$ system was able to induce lung fibrosis 14 days post-application (dpa), exhibiting an abnormal lung structure and elevated TGF $\beta 1$ levels (Appendix Fig S7A-C). Furthermore, nuclear fractionation from the AAV-TGF $\beta 1$ model showed increased nuclear actin levels (Appendix Fig S7D). Importantly, lung epithelial cells isolated from this model displayed high levels of ph-EZH2, phTAK1, actomyosin remodelling (marked by the phosphorylation of the myosin light chain, ph-MLC2) and POL2-K7m (Fig 7A), establishing the AAV-TGF $\beta 1$ as a suitable model for this study.

We next assessed whether interference with EZH2 activity in the epithelium would alter outcomes in injured lung and the suitability for pharmacological interventions. Since TGF $\beta 1$ levels in the bronchoalveolar lavage (BAL) fluid were increased rapidly within 3 days after AAV application compared to AAV-stuffer control vectors (Appendix Fig S7C), we thus chose to administer the EZH2 pharmacological inhibitor GSK126 (McCabe et al, 2012) one day post-AAV application in a prophylactic setting for a duration of 14 days. Consistent with our in vitro data, inhibition of EZH2 by GSK126 significantly blocked the phosphorylation of EZH2 on T311 and the methylation of POL2 on K7; however, it cannot attenuate ph-TAK1 as well as actomyosin remodelling (Fig 7A and Appendix Fig S7E). These data provide further evidence that TAK1 and actomyosin remodelling lie upstream of non-canonical EZH2 and support the relevance of our proposed model in vivo.

Importantly, the reduction in ph-EZH2 levels led to improved outcomes in the presence of fibrotic stimuli as shown by lung tissue structure (Appendix Fig S7F). Intriguingly, using an automatic unbiased deep learning approach for micro-computed tomography $(\mu \mathrm{CT})$ to analyse pathophysiological changes in the whole lung (Birk et al, 2020), we observed markedly improved lung architecture in the GSK126-exposed AAV-TGF $\beta 1$ mice (Fig 7B), correlating with decreased ph-EZH2 levels in epithelial cells (Appendix Fig S7G).

Figure 6. Actin dynamics regulate the profibrotic transcriptional response in injured AECs.

A Representative immunofluorescence images of F-actin (phalloidin) and DAPI show that treatment with ROCK inhibitor Y27632 but not depletion of EZH2 can prevent TGF $\beta 1$-induced actomyosin remodelling in AECs (scale bars $200 \mu \mathrm{m}$ ).

B Simple western analysis (Peggy Sue) of EZH2 immunoprecipitates shows abolition of TGF 31 -induced profibrotic transcriptional complex of EZH2/POL2/actin upon the convergent treatment of TGF 31 and Y27632. Unspecific IgG binding was used as a negative control. Representative from 3 biological replicates is shown.

C, D Simple western analysis (Peggy Sue) of nuclear fractionation (C) shows an increase in nuclear actin, ph-EZH2 and PO2-S2p levels in injured AECs. RNAi-mediated depletion of IPO9 (silPO9) prevents injury-induced nuclear actin and POL2-S2p. Quantification (D) shows mean + s.d. $\left(n=3\right.$ biological replicates, ${ }^{\star} P<0.05$, ${ }^{\star *} P<0.01,{ }^{* \star *} P<0.001$, ANOVA/Tukey's).

E $\quad$ QPCR analysis of profibrotic genes in MCS co-culture with AECs shows that depletion of IPO9 in TCF $\beta 1$-injured AECs blocks the fibrotic crosstalk with MCs. Data show mRNA levels of profibrotic genes normalised to $S 26$ (mean + s.d., $n=3$ biological replicates with 5 MCs donors, ${ }^{*} P<0.05$, ${ }^{\star \star \star} P<0.001$, ANOVA/Tukey's). 
A

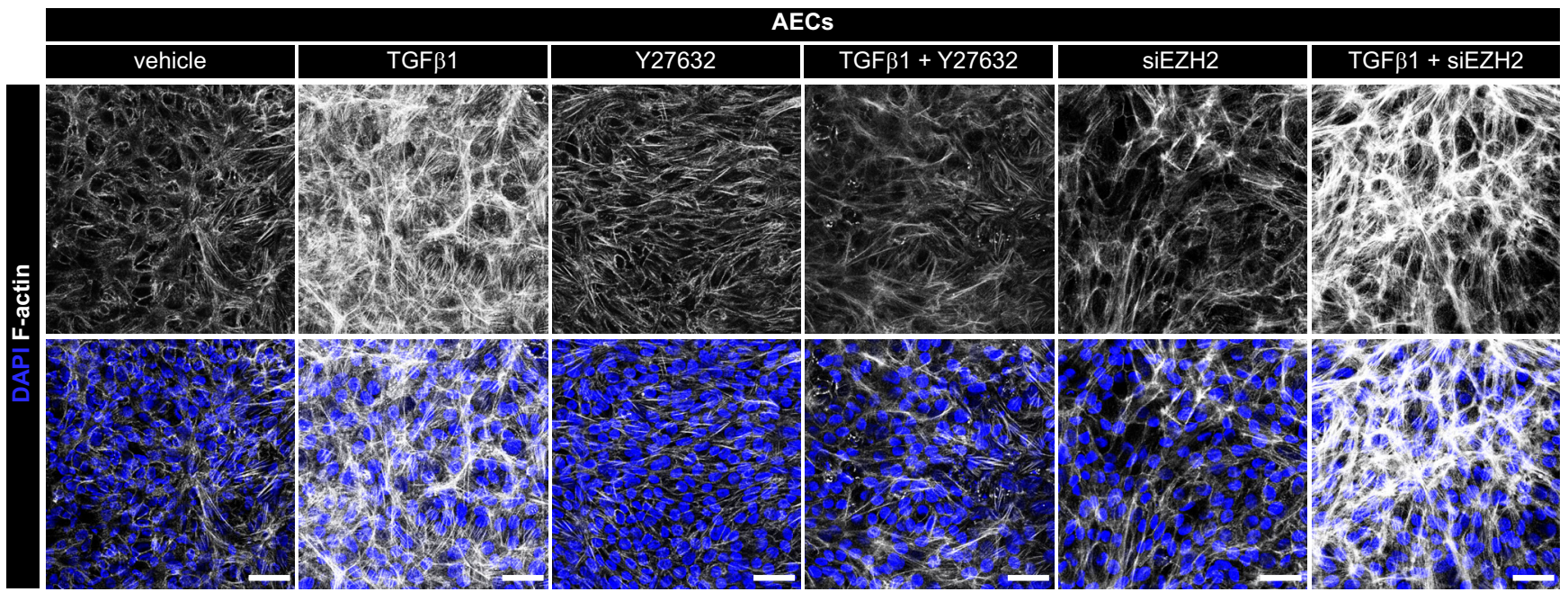

B

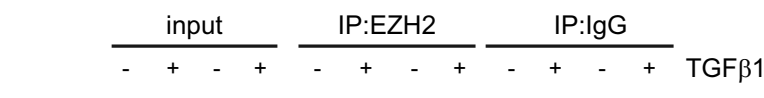

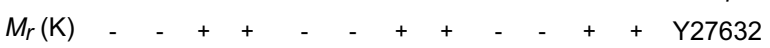

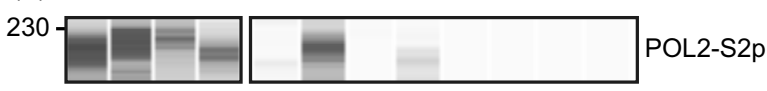

$116-2$ ph-EZH2

$40-=-2 \quad-\quad$ Actin

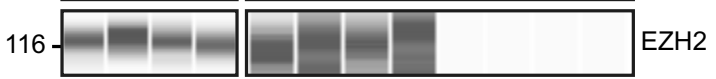

C

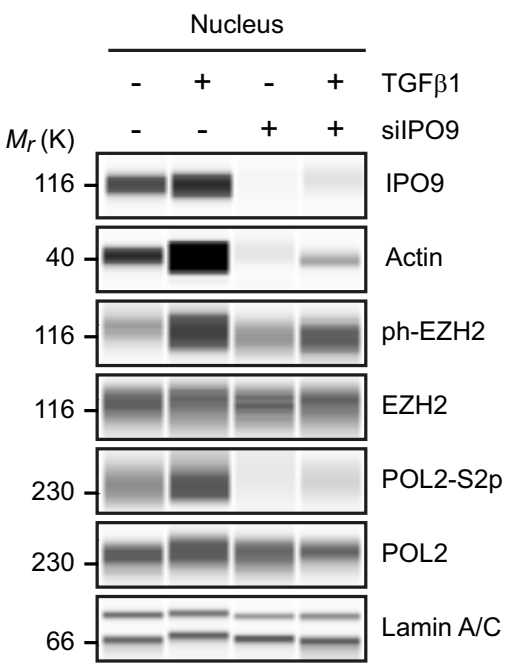

D

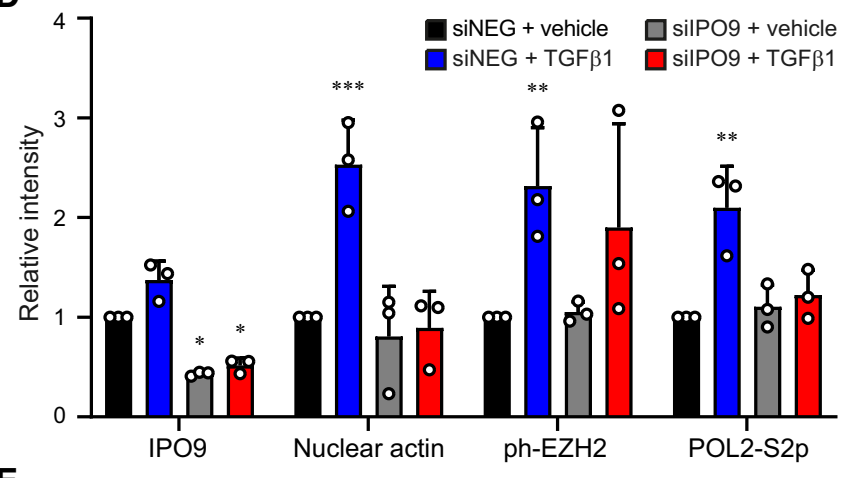

$\mathbf{E}$

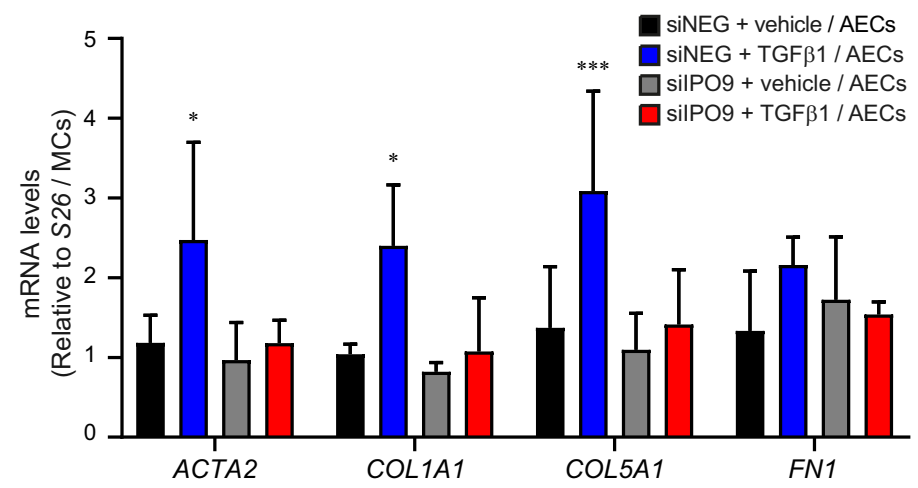

Figure 6.

Further analyses revealed reduced alveolar wall thickness (Appendix Fig S7H), less neutrophil influx in BAL and reduced MMP7 levels (a diagnostic biomarker for fibrosis) in the GSK126treated group (Appendix Fig S7I).

Bronchiolisation of the alveoli is an important feature of aberrant IPF repair, characterised by the presence of metaplastic keratin 5-positive $\left(\mathrm{KRT5}^{+}\right)$cells within the alveolar sacs to abandon the repair process of severely fibrotic tissue (Cassandras et al, 2020). The profibrotic nature of the EZH2-dependent transcription complex makes it a likely candidate behind alveolar bronchiolisation. Indeed, gene expression and protein analysis revealed a dramatic increase in the expression of $\mathrm{Krt5}$ and other fibrotic genes, whereas type I and type II alveolar cell markers (Pdpn and Sftpc, respectively) were decreased in the fibrotic epithelium (Fig 7C and D and Appendix Fig S7J). Importantly, these changes were EZH2-dependent as they were abolished by 
A
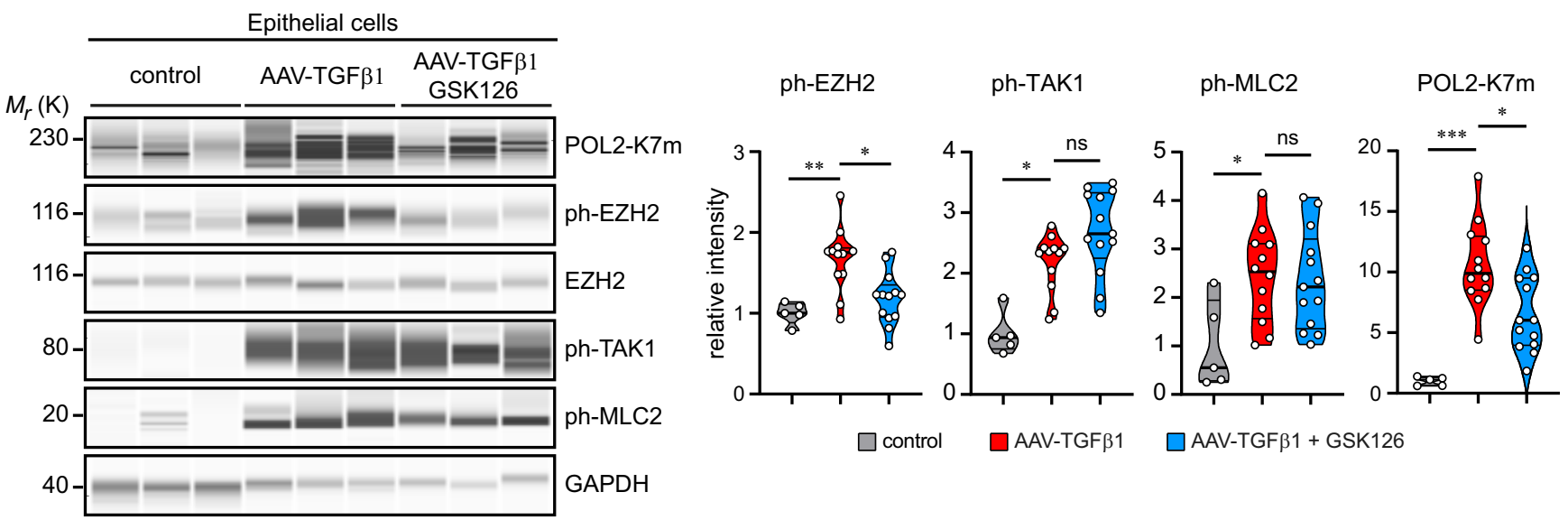

$\square$ control

$\square$ AAV-TGF $\beta 1$

$\square$ AAV-TGF $\beta 1+$ GSK126

B

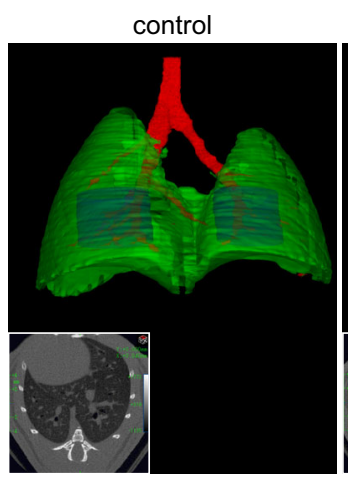

AAV-TGF $\beta 1$

AAV-TGF $\beta 1+$ GSK126

C
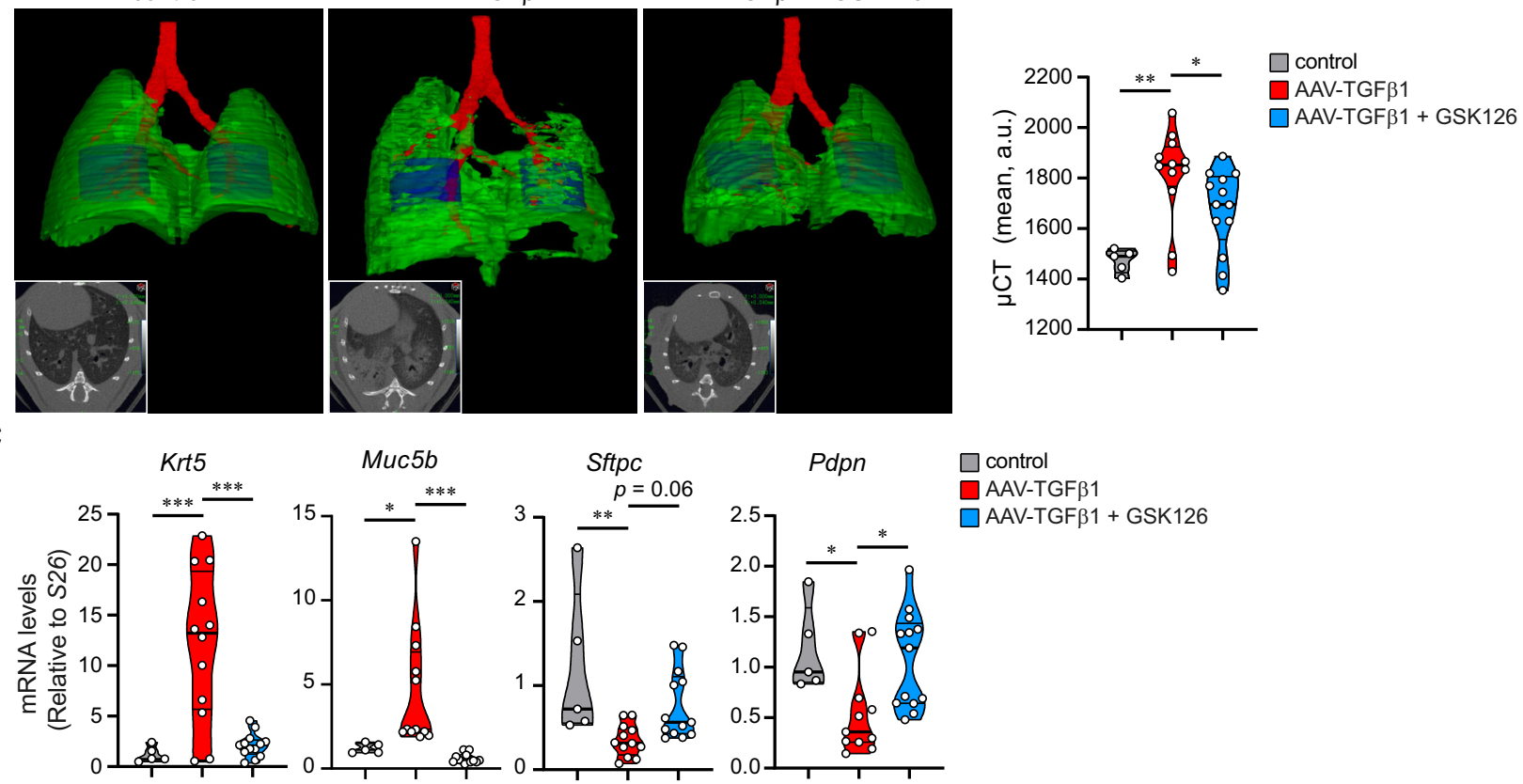

$\square$ control

$\square$ AAV-TGF $\beta 1$

$\square$ AAV-TGF $\beta 1+$ GSK126

D
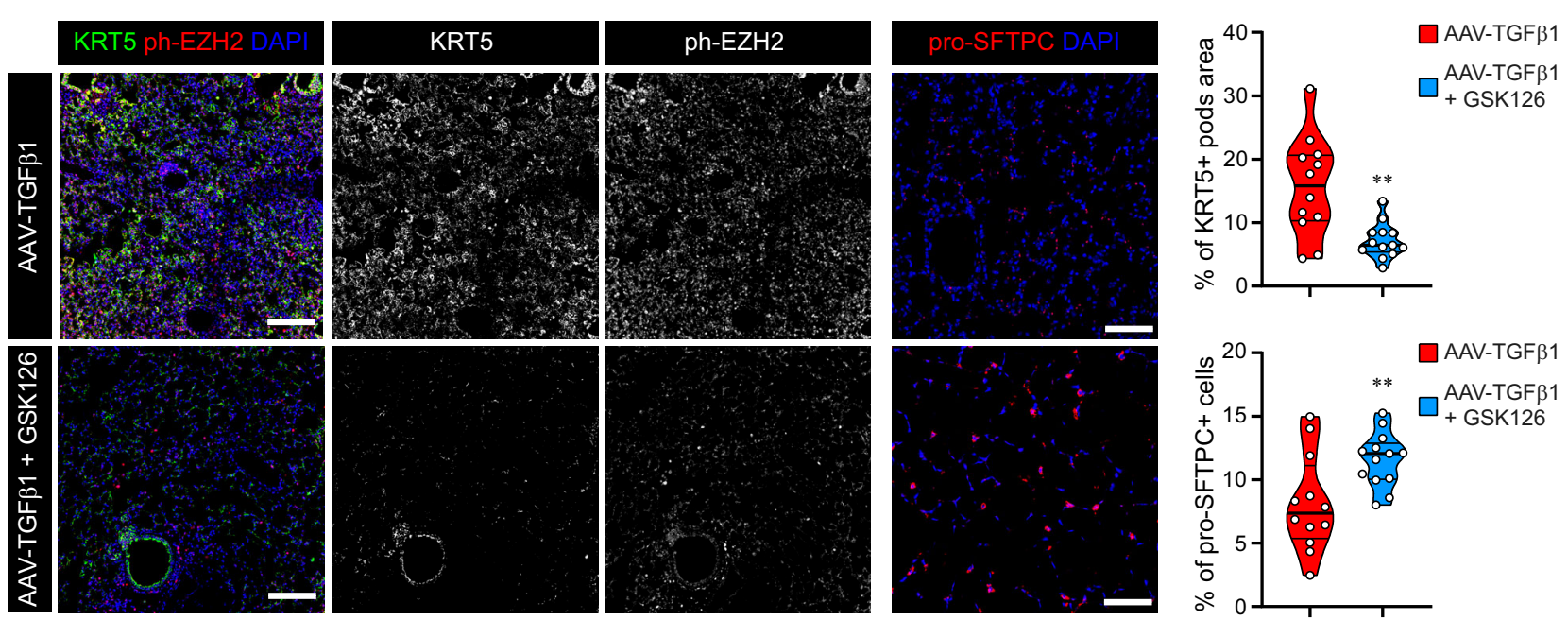

Figure 7. 
Figure 7. Non-canonical EZH2 regulates metaplastic differentiation of alveolar epithelium.

A Simple western analysis (Peggy Sue) and quantifications (right panels) of mouse lung epithelial cells shows increased ph-EZH2 (T311), ph-TAK1, myosin activity (phMLC2) and POL2-K7m levels in AAV-mediated TGF $\beta 1$ overexpression. Note, increased ph-EZH2 and POL2-K7m levels are attenuated by the EZH2 inhibitor GSK126, whereas ph-TAK1 and ph-MLC2 levels cannot be rescued by GSK126. Quantifications (right panels) show violin plots, ${ }^{\star} P<0.05,{ }^{* *} P<0.01,{ }^{\star * *} P<0.001$, ns $=$ nonsignificant, Kruskal-Wallis/Dunn's.

B Representative of 3D computed tomography (CT) reconstruction of the lung from control, AAV-TCF $\beta 1$ and GSK126-treated AAV-TCF $\beta 1$ mice (green: lung tissue, red: airways and region of interest (ROI): blue). Insets show $\mu \mathrm{CT}$ slices in the middle of the lung from respective mice. Note, GSK126 attenuates TCF $\beta 1$-induced lung injury. Quantification (right panel) shows mean intensity of ROIs from the whole lung (violin plots, ${ }^{\star} P=0.0385,{ }^{* *} P=0.0012, \mathrm{ANOVA} / \mathrm{Holm}-\mathrm{Sidak}$ 's).

C qPCR analysis of differentiation genes in epithelial cells reveals that EZH2 is required for the effect of TGF $\beta 1$ on metaplastic differentiation gene expression. Data show mRNA levels of profibrotic genes normalised to S26 (violin plots, ${ }^{*} P<0.05,{ }^{* \star} P<0.01$, ${ }^{* \star \star} P<0.001$, ANOVA/Holm-Sidak's).

D Immunofluorescence analysis of KRT5 as a marker for alveolar metaplastic basal cells and ph-EZH2 (scale bars $100 \mu \mathrm{m}$ ), pro-SFTPC as a marker for alveolar type 2 epithelial cells (scale bars $50 \mu \mathrm{m}$ ) and quantifications (right panels) show percentage of KRT5 ${ }^{+}$pods area per 10X field and percentage of pro-SFTPC ${ }^{+}$cells per 20X field $\left({ }^{*} P<0.05\right.$, unpaired $t$-test)

Data information: All violin plots display minimum, first quartile, median, third quartile and maximum; $n=5$ control, 12 AAV-TCF $\beta 1$ and 13 GSK126-treated AAV-TGF $\beta 1$ mice.

See also Appendix Fig S7.

Source data are available online for this figure.

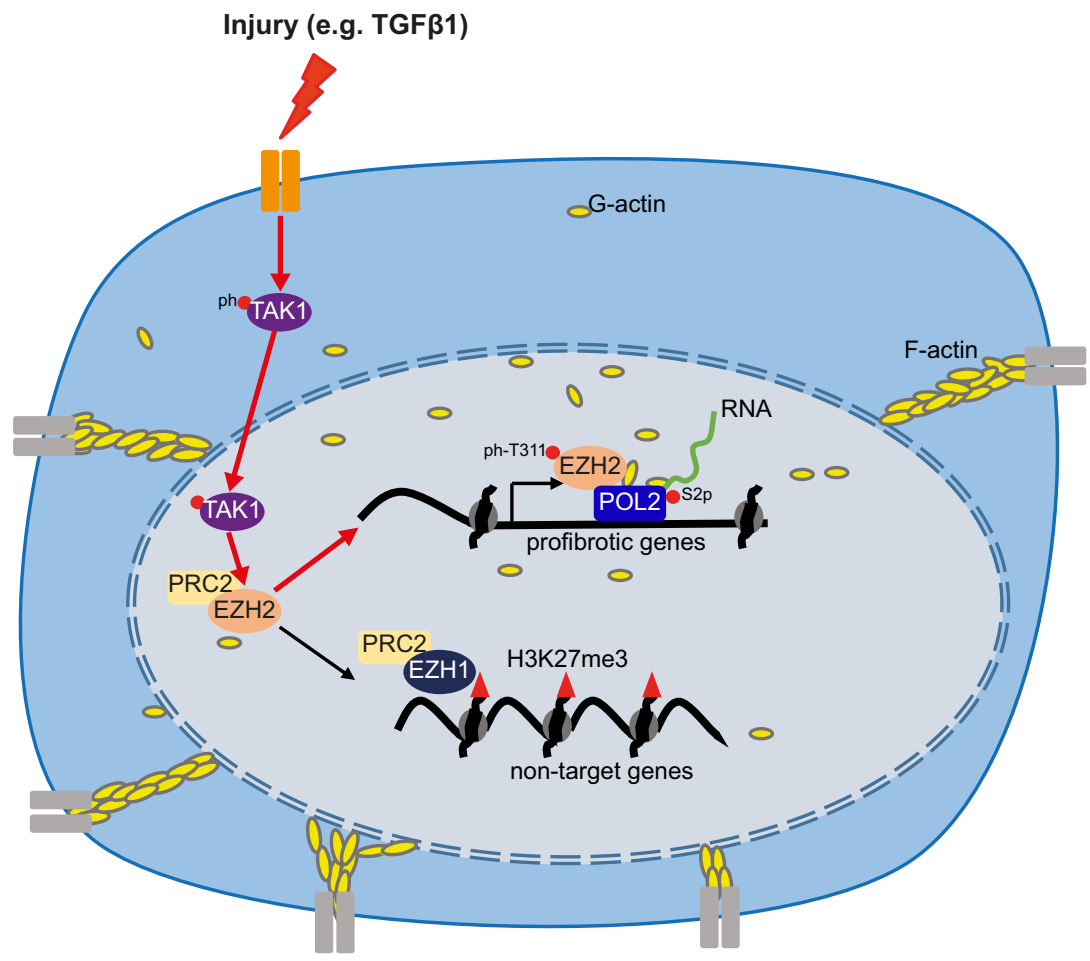

Figure 8. A model of injured respiratory epithelium initiating the profibrotic response through a fibrotic transcriptional complex of EZH2, POL2 and nuclear actin.

TCF $\beta 1$-injured epithelium activates TAK1 and actomyosin remodelling, which subsequently induces nuclear translocation of TAK1 and actin. Nuclear TAK1 mediates the phosphorylation of EZH2 on T311 and facilitates the release of EZH2 from PRC2. The liberation of EZH2 is accompanied by an EZ switch to EZH1-PRC2, which is required to maintain H3K27me3 at TCF $\beta 1$ non-target genes. Simultaneously, EZH2 establishes the fibrotic transcriptional complex with POL2 and nuclear actin to promote the metaplastic differentiation of AECs and triggers the fibrotic crosstalk with MCs. Perturbing this fibrotic complex blocks the fibrotic cascade, reinforces tissue repair and restores homeostasis.

GSK126. These experiments indicate that targeting EZH2 during injury can attenuate the progression of fibrosis and protect the lung structure and function from TGF $\beta 1$-induced fibrosis. Overall, our data demonstrate that non-canonical EZH2 signalling represents a novel therapeutic treatment for restoring homeostatic repair in the fibrotic lung.

\section{Discussion}

Our study provides direct evidence for the initiation of a fibrotic cascade downstream of epithelial injury, as summarised in Fig 8. We hereby propose a novel fibrotic model in which TGF $\beta 1$-injured lung epithelium promotes two mechanisms mediated by nuclear 
translocated TAK1/MAP3K7: (i) an EZ switch from EZH2-PRC2 to EZH1-PRC2, which is required to maintain silencing at non-fibrotic genes; and (ii) a PRC2-independent role of EZH2 that forms a profibrotic transcriptional complex including EZH2, POL2-S2p and nuclear actin to fine-tune the metaplastic transcriptional process.

Many studies have suggested the difference in the behaviour of MCs and IPF-MCs in vivo (Ramos et al, 2001; Huang et al, 2014; Habermann et al, 2020). However, such a difference in responsiveness has not been successfully demonstrated in vitro. We observed that IPF-MCs display a marked increase in FMT levels compared to healthy MCs when in co-culture with injured AECs, suggesting multifactorial stimuli from injury may activate MCs' epigenetic imprint of their origin (Ivanov et al, 2016; Shin et al, 2019). Furthermore, we observed a bidirectional fibrotic crosstalk between injured AECs and MCs, suggesting that successful repair of the fibrotic lung requires understanding the complex communications between different cell types within the lung. The exact molecular mechanisms underlying this process is an important open question for future studies.

POL2 has been reported to bind to EZH2-PRC2 at lineage-specific gene loci. This interaction establishes poised promoters that alter the chromatin state to ensure only strong and sustained signals are capable of driving transcription (Brookes et al, 2012). In our model, this process of transcriptional activation occurs in a stepwise fashion. Within the first $24 \mathrm{~h}$ of TGF $\beta 1$ treatment, the establishment of poised POL2 at profibrotic genes is induced, which could arise from canonical TGF $\beta 1 / \mathrm{SMAD}$ signalling (Zi et al, 2011). Consequently, accessibility to these genes is gradually increased, driving AECs to a plastic and inducible epigenetic state. Chronic exposure to TGF $\beta 1$ will then lead to the loss of PRC2 occupancy at these sites.

Importantly, we show an interaction between PRC2-independent EZH2 and the elongated form of POL2 to mediate profibrotic gene expression. This is an intriguing new function of EZH2 as a transcriptional activator in IPF. Another interesting observation is the increase in POL2-K7m modification in injured lung epithelial cells. This mark has been shown to be enriched at active promoters and associated with early transcription (Dias et al, 2015; Voss et al, 2015). Further studies should be directed towards better understanding this modification and its crosstalk with other modifications, such as S2p, S5p and K7 acetylation to fine-tune gene expression in development and disease.

The role of EZH2 in human diseases remains ambiguous; for example, elevated EZH2 levels correlate with disease progression and poor outcome in some patients (Tsou et al, 2019), while preventing the transition of disease in others (Wan et al, 2018; Grindheim et al, 2019). These opposing biological consequences likely depend on the specific disease state that leads to a chromatin landscape capable of activating PRC2 target genes (Laugesen \& Helin, 2014). This suggests EZH2 can drive cell fates in multiple directions, highlighting the importance of elucidating its roles in tissue-specific functions and disease states.

Interestingly, GSK126 is a S-adenosylmethionine competitor that selectively inhibits EZH2 methyltransferase activity (McCabe et al, 2012), suggesting that EZH2's enzymatic activity is required for its non-canonical function in driving aberrant epithelial remodelling. However, we cannot resolve all EZH2 substrate partners within this study. Future efforts should be directed towards the mechanistic action of EZH2 and its binding partners on fine-tuning the expression of profibrotic genes.
Remodelling of the actin cytoskeleton is a major pathway involved in TGF $\beta 1$ signalling (Rana et al, 2018). Our study supports this finding and provides further molecular detail on an actomyosindependent fibrotic cascade. TGF $\beta 1$ initiates epithelial injury in a non-mechanical manner; however, its effects are mechanically profound through aberrant physical influences such as tissue stiffness, altered cellular junctions and ECM deposition. Signalling to the nucleus through the actin dynamics directly relays mechanical forces onto the nucleus, thereby controlling transcriptional programmes (Le et al, 2016). Our results show that (i) blocking actomyosin remodelling or (ii) interference with nuclear actin influx is able to attenuate profibrotic gene expression. We hypothesise that alterations in mechanosensing may promote fibrosis and that regulation of profibrotic genes by non-canonical EZH2 is a potential mechanism. Our study has begun to dissect the molecular pathways that lead to non-canonical EZH2 function in fibrotic progression. Here, we show that injury induces nuclear translocation of activated TAK1, which phosphorylates EZH2 on T311 to facilitate the liberation of EZH2 from PRC2 and triggers fibrotic responses. It is noteworthy that targeting EZH2 in vivo led to improved outcomes in lung structure and function in the presence of a chronic fibrotic milieu, suggesting that targeting the TAK1-EZH2 axis might be a promising intervention for IPF and lung injury.

Taken together, our study sheds new light on the non-canonical functions of EZH2 as a transcriptional activator and thereby implies that enzymatic inhibitors of EZH2 may not be efficacious unless they are capable of disrupting its interaction with the transcriptional machinery, while maintaining the H3K27me3 occupancy at nontarget genes. However, current epigenetic inhibitors lack the ability to modify epigenetic states in a precise manner and the efficiency to target specific genomic loci (Roberti et al, 2019). We have therefore identified a novel profibrotic mechanism that could serve as a potential therapeutic target for the next generation of epigenetics therapeutics in IPF treatment.

\section{Materials and Methods}

\section{Primary cells and cell lines}

Human lung mesenchymal cells from healthy (Lonza, CC-2512) and IPF donors (IPF-MCs, Lonza, CC-7231) were grown in fibroblast growth medium-2 (Lonza, CC-3132). MCs from passages 4-8 were used. For comparison between healthy and IPF-derived MCs, MCs were used at passage 3. MCs were maintained on type I collagen (Corning, 354236) pre-coated cell culture flasks.

A549 alveolar epithelial cells (AECs, Sigma-Aldrich, 86012804) were grown in DMEM with $10 \%$ foetal calf serum (GIBCO). TGF $\beta$ reporter (MFB-F11) cell line was from Tony Wyss-Coray laboratory.

\section{AECs/MCs co-culture}

12,000 AECs were seeded on 6.5-mm Transwell (Corning, $0.4 \mu \mathrm{m}$ pore size, PET membrane, 3,470). 25,000 MCs per well (24-well plate, Corning, 3,526) were seeded on type I collagen (Corning) pre-coated plate. For comparison between healthy and IPF-derived MCs, MCs were seeded on $1 \mathrm{kPa}$ hydrogels (SS24-EC-1, Cell Guidance Systems) to mimic the lung stiffness. After reaching $50-70 \%$ 
confluency, cells were starved overnight, and medium was replaced by fresh DMEM with $50 \mu \mathrm{l}$ apical and $500 \mu \mathrm{l}$ basolateral before experiment start. The two cell type compartments were then brought together, and treatments were applied apically for $72 \mathrm{~h}$. For inhibitors experiments, AECs were pre-injured $24 \mathrm{~h}$ prior to treatment.

\section{RNA extraction and quality control}

After $72 \mathrm{~h}$ of TGF $\beta 1$ treatment in the co-culture, cells were lysed in RLT buffer supplemented with $1 \% \beta$-mercaptoethanol (QIAGEN). Total RNA was extracted and purified on the MagMAX instrument (Thermo Fisher), using the RNeasy 96 Kit \#74182 (QIAGEN) according to the manufacturer's instructions. Total RNA was quantitatively and qualitatively assessed using the fluorescence-based Broad Range Quant-iT RNA Assay Kit (Thermo Fisher) and the Standard Sensitivity RNA Analysis DNF-471 Kit on a 48-channel Fragment Analyzer (Agilent), respectively. Concentrations averaged at $25.4 \mathrm{ng} / \mu \mathrm{l}$ while RIN ranged from 9 to 10 , with a median of 10 .

\section{Whole transcriptome profiling with mRNA-seq}

Forty human epithelial cell- and mesenchymal cell-derived RNA samples were normalised on the MicroLab STAR automated liquid platform (Hamilton). Total RNA input of $200 \mathrm{ng}$ was used for library construction with the NEBNext Ultra II Directional RNA Library Prep Kit for Illumina \#E7760, together with the NEBNext Poly(A) mRNA Magnetic Isolation Module \#E7490 upstream and the NEXNext Multiplex Oligos for Illumina \#E7600 downstream (all New England Biolabs). The only deviation from the manufacturer's protocol was the use of Ampure XP beads (Beckman Coulter) for double-stranded cDNA purification, instead of the recommended SPRIselect Beads. The index PCR was performed with 12 cycles, while the final library elution was done in $35 \mu \mathrm{l}$. mRNA libraries were then quantified by the High Sensitivity dsDNA Quanti-iT Assay Kit (Thermo Fisher) on a Synergy HTX (BioTek). Library molarity averaged at $39 \mathrm{nM}$. mRNA libraries were also assessed for size distribution (smear analysis of $397 \mathrm{bp}$ average) and adapter dimer presence $(<0.5 \%)$ by the High Sensitivity Small Fragment DNF-477 Kit on a 48-channel Fragment Analyzer (Agilent). All 40 sequencing libraries were then normalised on the MicroLab STAR (Hamilton), pooled and spiked in with PhiX Control v3 (Illumina). The library pool was subsequently clustered on a Paired-End Flow Cell and sequenced on a HiSeq 4000 Sequencing System (Illumina) with dual index, paired-end reads at 75 bp length (Read parameters: Rd1: 76, Rd2: 8, Rd3: 8, Rd4: 76), reaching an average depth of 31 million Pass-Filter reads per sample $(7.9 \% \mathrm{CV})$.

\section{Bioinformatics analysis}

The processing pipeline was carried out as previously described (Sollner et al, 2017). Differentially expressed gene analysis was done using the limma R-package with Benjamini-Hochberg correction (Ritchie et al, 2015). Genes with an adjusted $P$ value $<0.05$ were considered significant.

Gene ontology term analyses were performed using Enrichr (Chen et al, 2013). Gene set enrichment analysis was performed on a pre-ranked gene list according to $\log _{2}$ fold change and compared with the Board Institute Molecular Database collection of chemical and genetic perturbations (C2 CGP, 3297 gene sets) and oncogenic signatures (C6, 189 gene sets) using the web-based tool available from the Broad Institute (Mootha et al, 2003; Subramanian et al, 2005). IPF molecular signature was derived from previous publications (Kusko et al, 2016; Habermann et al, 2020). In total, 18 different gene sets from epithelial, mesenchymal subpopulations and whole lung signature were used for this analysis.

\section{Mass spectrometry}

\section{Sample preparation}

Supernatant medium was buffer exchanged using 3.5-kDa Amicon filter (Millipore) to $8 \mathrm{M}$ urea, $50 \mathrm{mM}$ ammonium bicarbonate buffer. While in the filter, proteins were reduced with $5 \mathrm{mM}$ tris(2carboxyethyl)phosphine (TCEP) at $30^{\circ} \mathrm{C}$ for $60 \mathrm{~min}$ and subsequently alkylated with $15 \mathrm{mM}$ iodoacetamide (IAA) in the dark at room temperature for $30 \mathrm{~min}$. The buffer was then exchanged again to $1 \mathrm{M}$ urea, $50 \mathrm{mM}$ ammonium bicarbonate; the sample was recovered from the Amicon tube into a new microfuge tube, and protein concentration was determined using bicinchoninic acid (BCA) protein assay (Thermo Scientific). Proteins were subjected to overnight digestion with mass spec grade Trypsin/Lys-C mix (1:25 enzyme/substrate ratio). Following digestion, samples were acidified with formic acid (FA) and subsequently desalted using AssayMap C18 cartridges mounted on an Agilent AssayMap BRAVO liquid handling system. Cartridges were sequentially conditioned with $100 \%$ acetonitrile $(\mathrm{ACN})$ and $0.1 \% \mathrm{FA}$; samples were then loaded, washed with $0.1 \%$ FA and peptides eluted with $60 \%$ ACN, $0.1 \%$ FA. Finally, the organic solvent was removed in a SpeedVac concentrator prior to LC-MS/MS analysis.

\section{Mass spectrometry}

Prior to LC-MS/MS analysis, dried peptides were reconstituted with $2 \% \mathrm{ACN}, 0.1 \% \mathrm{FA}$, and concentration was determined using a NanoDrop $^{\mathrm{TM}}$ spectrophometer (Thermo Fisher). Samples were then analysed by LC-MS/MS using a Proxeon EASY-nanoLC system (Thermo Fisher) coupled to an Orbitrap Fusion Lumos mass spectrometer (Thermo Fisher Scientific). Peptides were separated using an analytical C18 Acclaim PepMap column $(75 \mu \mathrm{m} \times 500 \mathrm{~mm}$, $2 \mu \mathrm{m}$ particles; Thermo Scientific) at a flow rate of $300 \mathrm{nl} / \mathrm{min}$ $\left(60^{\circ} \mathrm{C}\right)$ using a 75 -min gradient: $1 \%$ to $5 \% \mathrm{~B}$ in $1 \mathrm{~min}, 6 \%$ to $23 \%$ $\mathrm{B}$ in $44 \mathrm{~min}, 23 \%$ to $34 \% \mathrm{~B}$ in $28 \mathrm{~min}$, and $34 \%$ to $48 \% \mathrm{~B}$ in 2 min (A $=$ FA $0.1 \%$; $\mathrm{B}=80 \%$ ACN: $0.1 \% \mathrm{FA}$ ). The mass spectrometer was operated in positive data-dependent acquisition mode. MS1 spectra were measured in the Orbitrap in a mass-tocharge $(\mathrm{m} / \mathrm{z})$ of $375-1,500$ with a resolution of 60,000 at $\mathrm{m} / \mathrm{z} 200$. Automatic gain control target was set to $4 \times 105$ with a maximum injection time of $50 \mathrm{~ms}$. The instrument was set to run in top speed mode with 2-s cycles for the survey and the MS/MS scans. After a survey scan, the most abundant precursors (with charge state between +2 and +7 ) were isolated in the quadrupole with an isolation window of $1.6 \mathrm{~m} / \mathrm{z}$ and fragmented with $\mathrm{HCD}$ at $30 \%$ normalised collision energy. Fragmented precursors were detected in the ion trap as rapid scan mode with automatic gain control target set to $1 \times 104$ and a maximum injection time set at $35 \mathrm{~ms}$. The dynamic exclusion was set to $20 \mathrm{~s}$ with a 10 ppm mass tolerance around the precursor. 


\section{MS data processing}

All raw files were processed with MaxQuant (Cox \& Mann, 2008; version 1.5.5.1) using the integrated Andromeda Search engine (Cox et al, 2011) against a target/decoy version of the curated human Uniprot proteome without isoforms (downloaded in January of 2019) and the GPM cRAP sequences (commonly known protein contaminants). First search peptide tolerance was set to $20 \mathrm{ppm}$; main search peptide tolerance was set to $4.5 \mathrm{ppm}$. Fragment mass tolerance was set to $20 \mathrm{ppm}$. Trypsin was set as enzyme in specific mode and up to two missed cleavages was allowed. Carbamidomethylation of cysteine was specified as fixed modification and protein $\mathrm{N}$-terminal acetylation and oxidation of methionine were considered variable modifications. The target-decoy-based false-discovery rate (FDR) filter for spectrum and protein identification was set to $1 \%$.

\section{Data analysis}

Quantitative analysis of the proteome data was performed in the $\mathrm{R}$ statistical programming language (version 3.5.1, 2018-07-02) using in-house R script wrapper for Bioconductor packages such as limma and MSstats. Briefly, feature (a peptide sequence of a given charge state and with potential amino acid modifications) intensities were $\log _{2}$-transformed and loess-normalised to account for systematic errors. Testing for differential abundance was performed using MSstats bioconductor package based on a linear mixed-effects model. In cases where a protein was completely missing in one of the conditions being compared, we imputed an empirical $\log _{2}$ fold change and $P$ value. Imputed $\log _{2}$ fold change was calculated by summing up the intensities of the protein across the replicates in the condition where it was detected, then dividing it by 3.3 and taking the logarithm base 2 of the number, whereas its imputed $p$ value was calculated by dividing 0.05 by the number of replicates the protein was detected.

\section{Chemical treatments}

Where indicated, AECs $24 \mathrm{~h}$ post-TGF $\beta 1$ application or MCs were treated with TGF $\beta 1$ inhibitor (ALK5i, Boehringer Ingelheim, EX76980, $0.2 \mu \mathrm{M}$ ), ROCK inhibitor (Y27632, Sigma-Aldrich, $10 \mu \mathrm{M}$ ), EZH2 inhibitor (GSK126, $10 \mathrm{nM}$ ), TAK1 inhibitor (5Z-7-Oxozeaenol; 5-OZ, Sigma-Aldrich, $1 \mu \mathrm{M}$ ), POL2-S2p inhibitor (5,6-Dichlorobenzimidazole 1- $\beta$-D-ribofuranoside; DRB, Sigma-Aldrich, $100 \mu \mathrm{M})$ and TGF $\beta 1$ (R\&D, 240-B, $1 \mathrm{ng} / \mathrm{ml}$ ). The vehicle dimethyl sulphoxide (DMSO) was used as control for ALK5, DRB, GSK126, Y27632 and 5-OZ treatments, $4 \mathrm{mM} \mathrm{HCl}$ (Sigma-Aldrich) containing $1 \mathrm{mg} / \mathrm{ml} \mathrm{BSA}$ (Sigma-Aldrich) was used as control for TGF $\beta 1$ treatment.

\section{Gene targeting}

siRNA targeting EZH2 (IDs: s4916 and s4918), IPO9 (IDs: s31299 and s31301), MAP3K7 (TAK1, IDs: s13766 and s13767) and negative control siRNA (AM4635) were from Invitrogen (Silencer Select). Transfection was performed 1 day before co-culture in AECs at $70 \%$ confluence, using Lipofectamine RNAiMAX (Invitrogen) according to the manufacturer's instruction. After $24 \mathrm{~h}$ of transfection, AECs were subjected to co-culture experiment.

sgRNA targeting EZH2 (ID: CRISPR687105_SGM) and negative control sgRNA (A35526) were from Invitrogen.
sgRNA targeting EZH2 was co-transfected with pEGFP-Puro vector (Addgene) using Lipofectamine CRISPRMAX Cas9 (Invitrogen) according to the manufacturer's instruction to AECs. The stable EZH2 knockout AECs were selected by Puromycin $(1.5 \mu \mathrm{g} / \mathrm{ml})$ and validated by qPCR and simple western analysis (Peggy Sue).

\section{Expression plasmids and transfection}

The EZH2 wildtype (EZH2-OHu27789C-pcDNA3.1-P2A-eGFP), EZH2-T311A and EZH2-T311D mutants were obtained from GenScript and transfected to stable EZH2 knockout AECs by Lipofectamine 3000 (Invitrogen) according to the manufacturer's instruction. One day after transfection, AECs were placed in co-culture with MCs for $72 \mathrm{~h}$.

\section{Co-immunoprecipitation (co-IP)}

Cells were harvested in IP lysis buffer (Thermo Fisher Scientific, 87788), containing protease and phosphatase inhibitors. Lysates were cleared by centrifugation at $10,000 \mathrm{~g}$ and protein concentrations were quantified with Pierce BCA (Thermo Fisher Scientific, 23225). Antibodies were coupled to protein A/G magnetic beads (Thermo Fisher Scientific, 26162) for $1 \mathrm{~h}$ at $4^{\circ} \mathrm{C} .500 \mu \mathrm{g}$ protein lysate was then incubated with $50 \mu$ beads-antibodies complex overnight at $4^{\circ} \mathrm{C}$. After repeated washing, proteins were eluted in NuPAGE LDS Sample buffer (Novex, NP0007) at $70^{\circ} \mathrm{C}$ in $10 \mathrm{~min}$, followed by simple western analysis (Peggy Sue).

Co-IP was performed using following antibodies: EZH2 (Active Motif, 39933, 1:100), POL2-S2p (Active Motif, 61083, 1:100), POL2K7m (Active Motif, 65691, 1:100) and SUZ12 (Cell Signalling, 3737, $1: 100$ ). An isotype IgG antibody (Cell Signaling, 2729S) was used as a negative control.

\section{In vitro kinase assay}

TAK1 in vitro kinase assays were carried out as previously described (Wan et al, 2018). In brief, recombinant human EZH2 protein (Novus Biologicals, H00002146-P01) was incubated with recombinant TAK1 protein (Novus Biologicals, H00006885-P01) in kinase buffer (Cell Signaling, 9802S), supplemented with $300 \mu \mathrm{M}$ ATP (Cell Signaling, 9804) for $60 \mathrm{~min}$ at $30^{\circ} \mathrm{C}$. The reaction was stopped by adding $5 \times$ fluorescent loading buffer, followed by WB analysis.

\section{Western blot (WB)}

Automatic WB system Peggy Sue, termed: simple western analysis (Peggy Sue), was carried out according to the manufacturer's instructions (Protein Simple, 043-225).

Classical WB (WB analysis) was carried out as previously described (Le et al, 2016), using Stella 3200 imaging system (Appendix Figs S3C and S5A).

The following antibodies were used: $\beta$-Actin (Cell Signaling, 3700), ph-AMPK $\alpha$ (Cell Signaling, 2535), AMPK $\alpha$ (Cell Signaling, 5831), EZH1 (Cell Signaling, 42088), EZH2 (Cell Signaling, 5246), EZH2 (Cell Signaling, 3147), ph-EZH2 (T311) (Cell Signaling, 27888), GAPDH (Cell Signaling, 3683), H3K27m3 (Cell Signaling, 9733), H3 (Cell Signaling, 4499), IPO9 (Invitrogen, PA5-25477), Lamin A/C (Cell Signaling, 4777), Lamin B1 (Cell Signaling, 17416), 
POL2 (Cell Signaling, 2629), POL2-S2p (Active Motif, 61083), POL2K7m (Active Motif, 65691), SUZ12 (Cell Signaling, 3737), TAK1 (Cell Signaling, 5206), ph-TAK1 (Cell Signaling, 4508), ph-MLC2 (Cell Signaling, 3674) and Cofilin (Cell Signaling, 5175).

\section{RT-qPCR}

RNA was isolated using the RNAeasy Plus Mini Kit (QIAGEN), after which reverse transcription was performed using the High-Capacity cDNA Reverse Transcription Kit (Bio-Rad). qPCR was performed on the ABI ViiA 7 real-time PCR System (Life Technologies) using TaqMan Fast Advanced Master Mix (Applied Biosytem, 4444557) with FAM- or VIC- labelled TaqMan MGB probe (Applied Biosystem) or DyNAmo ColorFlash SYBR Green (Thermo Scientific, F416L). Changes in gene expression were calculated using the comparative cycle threshold method with HPRT1, GAPDH or S26 as house-keeping controls. For a complete list of all predesigned probes (Life Technologies) and predesigned primers (Sigma-Aldrich, KSPQ12012G) used in this study see Table EV3.

\section{Chromatin immunoprecipitation (ChIP) analyses}

ChIP analyses were carried out as described previously (Le et al, 2016). In brief, cells were crosslinked in $1 \%$ formaldehyde (SigmaAldrich), after which cells were lysed and sonicated to fragment DNA. Following sonication, $10 \%$ was taken for input and lysates were incubated with $5 \mu \mathrm{g}$ antibody or isotype IgG overnight at $4^{\circ} \mathrm{C}$. The next day, lysates were incubated with protein A/G beads (Dynabeads, Life Technologies) followed by extensive washing. Chromatin was then decrosslinked and eluted. DNA was purified and analysed by qPCR. ChIP-qPCR was performed using DyNamo ColorFlash SYBR Green Mix (Thermo Fisher). Enrichment was determined by normalising to input DNA of the target gene as a percentage of input. For a complete list of primers, see Table EV3.

The following antibodies were used: EZH1 (Cell Signalling, 42088), EZH2 (Active Motif, 39933), ph-EZH2-T311 (Cell Signalling, 27888), H3K27m3 (Cell Signalling, 9733), POL2-S2p (Active Motif, 61083), POL2-S5p (Cell Signalling, 13523) and POL2 (Cell Signaling, 2629).

\section{ELISA}

The supernatant from the basolateral compartment of mono- and co-cultures or BAL fluid were harvested and human MMP7 (Meso Scale Discovery (MSD), F210K), mouse MMP7 (Aviva System Biology, OKCD05950), human MCP-1 (MSD, K151UGK), human Fibronectin (Thermo Fisher Scientific, BMS2028TEN), human TGF $\beta 1$ (R\&D, DY240), mouse TGFß1 (R\&D, DY1679) and COL1A1 (R\&D, DY6220) were performed according to the manufacturer's instructions. Cell lysates were extracted in RIPA buffer (Sigma-Aldrich, R0278) and E-cadherin (MSD, F21YX) or aSMA (custom-made, high binding plate from MSD, L15XB, with aSMA antibody from SigmaAldrich, A2547) was measured in accordance with the manufacturer's instructions. ELISA signal was measured either on a MESO SECTOR $^{\circledR} 6000$ using the MSD Discovery Workbench Software 4.0 (MSD, LLC., Rockville, MD, USA) or on a SpectraMaxM5 spectrophotometer (Molecular Devices) using SoftMax Pro 6.5 Software. Total protein concentration of the supernatant measured using the
Pierce BCA protein assay kit (Thermo Scientific) was used as a control.

\section{SEAP (secreted alkaline phosphatase) assay}

MFB-F11 cells were cultured as described previously (Tesseur et al, 2006). In brief, 20000 MFB-F11 cells were seeded per well in 96well plate until $70 \%$ confluency. Cells were washed twice with $1 \times$ PBS, followed by incubation with medium from the co-culture experiments for $24 \mathrm{~h}$ at $37^{\circ} \mathrm{C}, 5 \% \mathrm{CO}_{2}$. SEAP assay was carried out using Great EscAPe SEAP Chemiluminescence kit (Takara, 631738) according to the manufacturer's instruction.

\section{Immunofluorescence and confocal microscopy}

Cells were fixed in $4 \%$ paraformaldehyde or ice-cold methanol, permeabilised with $0.3 \%$ Triton X-100 in PBS and blocked in 5\% bovine serum albumin (BSA). Samples were incubated overnight in primary antibody followed by washing and incubation in secondary antibody and/or phalloidin labelling. Finally, samples were mounted with Prolong Antifade Mountant with DAPI (Invitrogen P36935).

For staining of lung sections, fixed specimen was dehydrated using Tissue Tek VIP processor and embedded into paraffin blocks. $3 \mu \mathrm{m}$ sections were deparaffinised by xylene and rehydrated by series of ethanol. Antigen retrieval was performed with sodium citrate buffer at $95^{\circ} \mathrm{C}$ for $30 \mathrm{~min}$. Autofluorescence was quenched by TrueVIEW Autofluorescence Quenching Kit (Vector Laboratories, SP-8400-15). Sections were blocked with $10 \%$ goat serum (SigmaAldrich) at room temperature for $1 \mathrm{~h}$, followed by incubation with primary and secondary antibodies. Samples were then mounted with Prolong Antifade Mountant.

The following primary antibodies were used: aSMA (SigmaAldrich A2547; 1:500), E-cadherin (BD 310181; 1:500), collagen type I (Sigma-Aldrich SAB4200678; 1:300), H3K27me3 (Cell Signaling 9733; 1:500), EZH2 (Cell Signaling 3147; 1:500), ph-EZH2-T311 (Cell Signaling 27888; 1:300), pro-SFTPC (Millipore, AB3786, 1:500), PDPN (BioLegend, 127401, 1:500) and KRT5 (Progen, GP-CK5, $1: 500)$.

All fluorescence images were collected by laser scanning confocal microscopy (LSM710; Zeiss) with an AXIO Observer Z1 using $20 \times, 40 \times$ or $63 \times$ immersion objectives. Regions of interest were randomly selected on the basis of DAPI staining. Images were captured with the same settings for control and treated samples. All image analyses were carried out using ImageJ software.

\section{Histology}

Haematoxylin-eosin staining of paraffin-embedded SAEC sections was performed using standard protocols. Images were collected with a Zeiss Axio Observer system using a $20 \times$ and $40 \times$ objectives.

\section{AAV-TGFB1-induced pulmonary fibrosis model}

The AAV6.2-stuffer and AAV6.2-TGF $\beta 1$ were produced and applied as previously described (Strobel et al, 2015; Strobel et al, 2019). In brief, AAV application was performed using male C57BL/6JRj mice (10-12 weeks, Janvier Labs) and housed in specific pathogen-free 
conditions. We used 5 mice for AAV-stuffer and 28 mice for AAVTGF $\beta 1$ treatment for a duration of 14 days. Sedated mice (by $3-4 \%$ isoflurane, Abbott) were administered intratracheally to the whole lung with a single dose of $50 \mu$ of viral vector suspension per mouse using a 22G Braunüle cannula (Vasofix, 4268091B). AAV doses are $2.5 \times 10^{11}$ vector genomes per animal. Where indicated, 14 mice from the AAV-TGF $\beta 1$ group received every day (except weekends) intraperitoneal injections of $150 \mathrm{mg} / \mathrm{kg}$ GSK126 for 14 days (Boehringer Ingelheim, EX00101320) in 20\% Captisol (Activate Scientific $\mathrm{GmbH}$, AS90498), one day post-AAV application. The other 14 mice in the AAV-TGF $\beta 1$ group received identical volume of $20 \%$ Captisol daily as a negative control group. Two mice in AAVTGF $\beta 1$ group and 1 mouse from GSK126 group died during treatment. All animal experiments were performed according to the German law on animal welfare and approved by the Regierungspräsidium Tübingen Nr. 16-028-03. No statistical method was used to pre-determine sample size.

For bronchoalveolar lavage fluid, lungs were washed twice with $0.7 \mathrm{ml}$ of Hank's balanced salt solution (Gibco, 14170-138), supplemented with $0.6 \mu \mathrm{M}$ EDTA (Invitrogen, AM9260G) and 1\% BSA (Sigma-Aldrich, A9576).

Ashcroft fibrosis score was performed on haematoxylin and eosin sections by a blinded pathologist according to the established guidelines (Heinemann et al, 2018).

\section{Micro-computed tomography}

$\mu \mathrm{CT}$ imaging procedure and analysis were performed as previously described (Birk et al, 2020). Briefly, lung images from sedated mice were performed using a Quantum FX $\mu \mathrm{CT}$ scanner (PerkinElmer). The lungs were scanned $360^{\circ}$ (512 slices of $0.4 \times 0.4 \times 0.4$ voxel size) to capture the entire lung. Threshold-based, automated segmentation and generation of lung densitometric data were performed using Analyze 12 (AnalyzeDirect).

\section{Magnetic cell separation}

Lung tissue was homogenised by the gentleMACS Dissociator (Miltenyi Biotec) using a standard lung dissociation kit (Miltenyi Biotec, 130-095-927) according to the manufacturer's protocol. Leucocyte subtypes were depleted by using CD45 MicroBeads (Miltenyi Biotec, 130-052-301), and dead cells were removed by Dead Cell Removal Kit (Miltenyi Biotec, 130-090-101). Epithelial cells were subsequently enriched by CD326 MicroBeads (EpCAM ${ }^{+}$, Miltenyi Biotec, 130-105-958) using a magnetic column and lysed for RNA and protein isolation.

\section{Statistical analysis and reproducibility}

Statistical analyses were performed using GraphPad Prism (version 8.0). Statistical significance was determined by the Mann-Whitney $U$-test, Wilcoxon test, paired $t$-test, Kruskal-Wallis test, repeated measurement or ordinary ANOVA with Dunn's, Tukey's or Dunnett's post hoc test, nonlinear/linear regression, Spearman's or Pearson's rank correlation coefficient test as indicated in the corresponding figure legends. In all comparisons which a test for normally distributed data was used, Gaussian distribution was determined with Kolmogorov-Smirnov test $(\alpha=0.05)$. All experiments in this study were repeated for at least three biological replicates.

\section{Data availability}

RNA-seq data that support this study have been deposited in the Gene Expression Omnibus GSE155832 (http://www.ncbi.nlm.nih. gov/geo/query/acc.cgi?acc $=$ GSE155832) $\quad($ GEO Accession viewer $)$ and the secretomics data in the proteomics repository ProteomeXchange Dataset PXD025677 (http://proteomecentral.proteomexcha nge.org/cgi/GetDataset?ID = PXD025677). All other data that support the finding are available from the authors on request.

Expanded View for this article is available online.

\section{Acknowledgements}

We thank S. A. Wickström, J. Morgner, C.Y.C. Yeung and members of lung repair and regeneration department for critical reading of the manuscript, G. Birk for $\mu \mathrm{CT}$ imaging analysis, B. Stierstorfer for generating Ashcroft score and technical assistance, J., Blender, J. Schütt and E. Peter for technical assistance, and Drug Discovery Sciences department - Boehringer Ingelheim Pharma GmbH \& Co. KG for technical support. This work is funded by Boehringer Ingelheim Pharma $\mathrm{GmbH} \&$ Co. KG and UK Medical Research Foundation Fellowship (MRF-091-0001-RG-GARNE to J.P.G.).

\section{Author contributions}

HQL and JPG conceived and supervised the study. HQL and MAH designed, performed and analysed most of the experiments. HQL, KQ, DK and CV performed and analysed RNA-seq experiments. HQL, ARC and JL performed and analysed secretomics experiments. MK and HS performed in vivo experiments. IK, WS-W, VS, JW, ES and BS performed experiments. JPG, FEH and MJT assisted with initial design and concept for the co-culture model. DL provided conceptual advice and supervised in vivo experiments. HQL and JPG provided conceptual advice. HQL, MAH and JPG wrote the paper. All authors commented on and edited the manuscript.

\section{Conflict of interest}

H.Q.L., M.A.H., I.K., M.K., V.S., J.W., W.S., E.S., K.Q., D.K., C.V., B.S., H.S., F.E.H., M.J.T., D.L. and J.P.G are employees of Boehringer Ingelheim $\mathrm{GmbH}$ \&Co. KG., J.L. is employee of Boehringer Ingelheim Pharmaceuticals, Inc., and A.R.C. is funded by Boehringer Ingelheim Pharmaceuticals, Inc.

\section{References}

Andrews D, Oliviero G, De Chiara L, Watson A, Rochford E, Wynne K, Kennedy C, Clerkin S, Doyle B, Godson C et al (2019) Unravelling the transcriptional responses of TGF-beta: Smad3 and EZH2 constitute a regulatory switch that controls neuroretinal epithelial cell fate specification. FASEB J 33: $6667-6681$

Aoyama K, Oshima M, Koide S, Suzuki E, Mochizuki-Kashio M, Kato Y, Tara S, Shinoda D, Hiura N, Nakajima-Takagi Y et al (2018) Ezh1 targets bivalent genes to maintain self-renewing stem cells in Ezh2-insufficient myelodysplastic syndrome. iscience 9: 161-174.

Birk G, Kastle M, Tilp C, Stierstorfer B, Klee S (2020) Automatization and improvement of muCT analysis for murine lung disease models using a deep learning approach. Respir Res 21: 124 
Boesch M, Baty F, Brutsche MH, Tamm M, Roux J, Knudsen L, Gazdhar A, Geiser T, Khan P, Hostettler KE (2020) Transcriptomic profiling reveals disease-specific characteristics of epithelial cells in idiopathic pulmonary fibrosis. Respir Res 21: 165

Bracken AP, Dietrich N, Pasini D, Hansen KH, Helin K (2006) Genome-wide mapping of Polycomb target genes unravels their roles in cell fate transitions. Genes Deu 20: 1123-1136

Brookes E, de Santiago I, Hebenstreit D, Morris K, Carroll T, Xie S, Stock J, Heidemann M, Eick D, Nozaki N et al (2012) Polycomb associates genomewide with a specific RNA polymerase II variant, and regulates metabolic genes in ESCs. Cell Stem Cell 10: 157-170

Cassandras M, Wang C, Kathiriya J, Tsukui T, Matatia P, Matthay M, Wolters P, Molofsky A, Sheppard D, Chapman H et al (2020) Gli1(+) mesenchymal stromal cells form a pathological niche to promote airway progenitor metaplasia in the fibrotic lung. Nat Cell Biol 22: 1295-1306

Chen EY, Tan CM, Kou Y, Duan Q, Wang Z, Meirelles CV, Clark NR, Ma'ayan A (2013) Enrichr: interactive and collaborative HTML5 gene list enrichment analysis tool. BMC Bioinformatics 14: 128

Cox J, Mann M (2008) MaxQuant enables high peptide identification rates, individualized p.p.b.-range mass accuracies and proteome-wide protein quantification. Nat Biotechnol 26: 1367-1372

Cox J, Neuhauser N, Michalski A, Scheltema RA, Olsen JV, Mann M (2011) Andromeda: a peptide search engine integrated into the MaxQuant environment. J Proteome Res 10: 1794-1805

Cruz-Molina S, Respuela P, Tebartz C, Kolovos P, Nikolic M, Fueyo R, van ljcken WFJ, Grosveld F, Frommolt P, Bazzi H et al (2017) PRC2 facilitates the regulatory topology required for poised enhancer function during pluripotent stem cell differentiation. Cell Stem Cell 20: $689-705$

Dias JD, Rito T, Torlai Triglia E, Kukalev A, Ferrai C, Chotalia M, Brookes E, Kimura H, Pombo A (2015) Methylation of RNA polymerase II nonconsensus Lysine residues marks early transcription in mammalian cells. Elife 4: e11215

Dopie J, Skarp KP, Rajakyla EK, Tanhuanpaa K, Vartiainen MK (2012) Active maintenance of nuclear actin by importin 9 supports transcription. Proc Natl Acad Sci USA 109: E544-E552

Fernandez IE, Eickelberg O (2012) The impact of TGF-beta on lung fibrosis: from targeting to biomarkers. Proc Am Thorac Soc 9: 111-116

Fraser NW, Sehgal PB, Darnell JE (1978) DRB-induced premature termination of late adenovirus transcription. Nature 272: 590-593

Grindheim JM, Nicetto D, Donahue G, Zaret KS (2019) Polycomb repressive complex 2 proteins EZH1 and EZH2 regulate timing of postnatal hepatocyte maturation and fibrosis by repressing genes with euchromatic promoters in mice. Gastroenterology 156: 1834-1848

Habermann AC, Gutierrez AJ, Bui LT, Yahn SL, Winters NI, Calvi CL, Peter L, Chung M-I, Taylor CJ, Jetter $C$ et al (2020) Single-cell RNA sequencing reveals profibrotic roles of distinct epithelial and mesenchymal lineages in pulmonary fibrosis. Sci Adv 6: eaba1972

Heinemann F, Birk G, Schoenberger T, Stierstorfer B (2018) Deep neural network based histological scoring of lung fibrosis and inflammation in the mouse model system. PLoS One 13: e0202708

Hewlett JC, Kropski JA, Blackwell TS (2018) Idiopathic pulmonary fibrosis: Epithelial-mesenchymal interactions and emerging therapeutic targets. Matrix Biol 71-72: 112-127

Huang SK, Scruggs AM, McEachin RC, White ES, Peters-Golden M (2014) Lung fibroblasts from patients with idiopathic pulmonary fibrosis exhibit genome-wide differences in DNA methylation compared to fibroblasts from nonfibrotic lung. PLoS One 9: e107055
Iriondo O, Liu Y, Lee G, Elhodaky M, Jimenez C, Li L, Lang J, Wang P, Yu M (2018) TAK1 mediates microenvironment-triggered autocrine signals and promotes triple-negative breast cancer lung metastasis. Nat Commun 9: 1994

Ivanov NA, Tao R, Chenoweth JG, Brandtjen A, Mighdoll MI, Genova JD, McKay RD, Jia Y, Weinberger DR, Kleinman JE et al (2016) Strong components of epigenetic memory in cultured human fibroblasts related to site of origin and donor age. PLoS Genet 12: e1005819

Kim KK, Sheppard D, Chapman HA (2018) TGF-beta1 signaling and tissue fibrosis. Cold Spring Harb Perspect Biol 10: a022293

Kusko RL, Brothers JF, Tedrow J, Pandit K, Huleihel L, Perdomo C, Liu G, JuanGuardela B, Kass D, Zhang $S$ et al (2016) Integrated genomics reveals convergent transcriptomic networks underlying chronic obstructive pulmonary disease and idiopathic pulmonary fibrosis. Am J Respir Crit Care Med 194: $948-960$

Laugesen A, Helin K (2014) Chromatin repressive complexes in stem cells, development, and cancer. Cell Stem Cell 14: 735-751

Lavarone E, Barbieri CM, Pasini D (2019) Dissecting the role of H3K27 acetylation and methylation in PRC2 mediated control of cellular identity. Nat Commun 10: 1679

Le HQ, Ghatak S, Yeung CY, Tellkamp F, Gunschmann C, Dieterich C, Yeroslaviz A, Habermann B, Pombo A, Niessen CM et al (2016) Mechanical regulation of transcription controls Polycomb-mediated gene silencing during lineage commitment. Nat Cell Biol 18: 864-875

Lu C, Sidoli S, Kulej K, Ross K, Wu CH, Garcia BA (2019) Coordination between TCF-beta cellular signaling and epigenetic regulation during epithelial to mesenchymal transition. Epigenetics Chromatin 12: 11

McCabe MT, Ott HM, Ganji G, Korenchuk S, Thompson C, Van Aller CS, Liu Y, Graves AP, lii ADP, Diaz E et al (2012) EZH2 inhibition as a therapeutic strategy for lymphoma with EZH2-activating mutations. Nature 492: $108-112$

Meissner A, Mikkelsen TS, Gu H, Wernig M, Hanna J, Sivachenko A, Zhang X, Bernstein BE, Nusbaum C, Jaffe DB et al (2008) Genome-scale DNA methylation maps of pluripotent and differentiated cells. Nature 454: $766-770$

Mootha VKLC, Eriksson KF, Subramanian A, Sihag S, Lehar J, Puigserver P, Carlsson E, Ridderstråle M, Laurila E, Houstis N et al (2003) PGC-1alpharesponsive genes involved in oxidative phosphorylation are coordinately downregulated in human diabetes. Nat Genet 34: 267-273

Okuda S, Takata N, Hasegawa Y, Kawada M, Inoue Y, Adachi T, Sasai Y, Eiraku M (2018) Strain-triggered mechanical feedback in self-organizing opticcup morphogenesis. Sci Adv 4: 12

Pasini D, Bracken AP, Hansen JB, Capillo M, Helin K (2007) The polycomb group protein Suz12 is required for embryonic stem cell differentiation. Mol Cell Biol 27: 3769-3779

Ramos C, Montaño M, Garcia-Alvarez J, Ruiz V, Uhal BD, Selman M, Pardo A (2001) Fibroblasts from idiopathic pulmonary fibrosis and normal lungs differ in growth rate, apoptosis, and tissue inhibitor of metalloproteinases expression. Am J Respir Cell Mol Biol 24: 591-598

Rana MK, Aloisio FM, Choi C, Barber DL (2018) Formin-dependent TGF-beta signaling for epithelial to mesenchymal transition. Mol Biol Cell 29: $1465-1475$

Ritchie ME, Phipson B, Wu D, Hu Y, Law CW, Shi W, Smyth GK (2015) limma powers differential expression analyses for RNA-sequencing and microarray studies. Nucleic Acids Res 43: e47

Roberti A, Valdes AF, Torrecillas R, Fraga MF, Fernandez AF (2019) Epigenetics in cancer therapy and nanomedicine. Clin Epigenetics 11: 81

Roth GJ, Heckel A, Brandl T, Grauert M, Hoerer S, Kley JT, Schnapp G, Baum P, Mennerich D, Schnapp A et al (2010) Design, synthesis, and evaluation of 
indolinones as inhibitors of the transforming growth factor beta receptor (TGFbetaRI). J Med Chem 53: 7287-7295

Sakai N, Tager AM (2013) Fibrosis of two: Epithelial cell-fibroblast interactions in pulmonary fibrosis. Biochim Biophys Acta 1832: 911-921

Sasai Y (2013) Cytosystems dynamics in self-organization of tissue architecture. Nature 493: 318-326

Selman M, Pardo A (2020) The leading role of epithelial cells in the pathogenesis of idiopathic pulmonary fibrosis. Cell Signal 66: 109482

Shin JY, Beckett JD, Bagirzadeh R, Creamer TJ, Shah AA, McMahan Z, Paik JJ, Sampedro MM, MacFarlane EG, Beer MA et al (2019) Epigenetic activation and memory at a TCFB2 enhancer in systemic sclerosis. Sci Transl Med 11: eaaw0790

Skourti-Stathaki K, Torlai Triglia E, Warburton M, Voigt P, Bird A, Pombo A (2019) R-Loops Enhance Polycomb Repression at a Subset of Developmental Regulator Genes. Mol Cell 73: 930-945

Sollner JF, Leparc G, Hildebrandt T, Klein H, Thomas L, Stupka E, Simon E (2017) An RNA-Seq atlas of gene expression in mouse and rat normal tissues. Sci Data 4: 170185

Strobel B, Duechs MJ, Schmid R, Stierstorfer BE, Bucher H, Quast K, Stiller D, Hildebrandt T, Mennerich D, Gantner F et al (2015) Modeling pulmonary disease pathways using recombinant adeno-associated virus 6.2. Am J Respir Cell Mol Biol 53: 291-302

Strobel B, Zuckschwerdt K, Zimmermann G, Mayer C, Eytner R, Rechtsteiner P, Kreuz S, Lamla T (2019) Standardized, scalable, and timely flexible adeno-associated virus vector production using frozen high-density HEK293 cell stocks and CELLdiscs. Hum Gene Ther Methods 30: 23-33

Subramanian A, Tamayo P, Mootha VK, Mukherjee S, Ebert BL, Gillette MA, Paulovich A, Pomeroy SL, Golub TR, Lander ES et al (2005) Gene set enrichment analysis: a knowledge-based approach for interpreting genome-wide expression profiles. Proc Natl Acad Sci USA 102: $15545-15550$

Suh H, Ficarro SB, Kang UB, Chun Y, Marto JA, Buratowski S (2016) Direct analysis of phosphorylation sites on the Rpb1 C-terminal domain of RNA polymerase II. Mol Cell 61: 297-304

Tesseur I, Zou K, Berber E, Zhang H, Wyss-Coray T (2006) Highly sensitive and specific bioassay for measuring bioactive TCF-beta. BMC Cell Biol 7: 15
Tsou PS, Campbell P, Amin MA, Coit P, Miller S, Fox DA, Khanna D, Sawalha AH (2019) Inhibition of EZH2 prevents fibrosis and restores normal angiogenesis in scleroderma. Proc Natl Acad Sci USA 116: 3695-3702

Voss K, Forné I, Descostes N, Hintermair C, Schüller R, Maqbool MA, Heidemann M, Flatley A, Imhof A, Gut M et al (2015) Site-specific methylation and acetylation of lysine residues in the $\mathrm{C}$-terminal domain (CTD) of RNA polymerase II. Transcription 6: 91-101

Wan L, Xu K, Wei Y, Zhang J, Han T, Fry C, Zhang Z, Wang YV, Huang L, Yuan $M$ et al (2018) Phosphorylation of EZH2 by AMPK suppresses PRC2 methyltransferase activity and oncogenic function. Mol Cell 69: 279-291

Wei M, Fan X, Ding M, Li R, Shao S, Hou Y, Meng S, Tang F, Li C, Sun Y (2020) Nuclear actin regulates inducible transcription by enhancing RNA polymerase II clustering. Sci Adu 6: eaay6515

Wickstrom SA, Niessen CM (2018) Cell adhesion and mechanics as drivers of tissue organization and differentiation: local cues for large scale organization. Curr Opin Cell Biol 54: 89-97

Xu K, Wu ZJ, Groner AC, He HH, Cai C, Lis RT, Wu X, Stack EC, Loda M, Liu T et al (2012) EZH2 oncogenic activity in castration-resistant prostate cancer cells is polycomb-independent. Science 338: 1465-1469

Yan J, Li B, Lin B, Lee PT, Chung T-H, Tan J, Bi C, Lee XT, Selvarajan V, Ng S-B et al (2016) EZH2 phosphorylation by JAK3 mediates a switch to noncanonical function in natural killer/T-cell lymphoma. Blood 128: 948-958

Zepp JA, Morrisey EE (2019) Cellular crosstalk in the development and regeneration of the respiratory system. Nat Reu Mol Cell Biol 20: 551-566

Zepp JA, Zacharias WJ, Frank DB, Cavanaugh CA, Zhou S, Morley MP, Morrisey EE (2017) Distinct mesenchymal lineages and niches promote epithelial self-renewal and Myofibrogenesis in the lung. Cell 170: 1134-1148

Zi Z, Feng Z, Chapnick DA, Dahl M, Deng D, Klipp E, Moustakas A, Liu X (2011) Quantitative analysis of transient and sustained transforming growth factor-beta signaling dynamics. Mol Syst Biol 7: 492

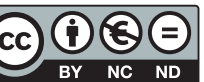

License: This is an open access article under the terms of the Creative Commons Attribution-NonCom mercial-NoDerivs License, which permits use and distribution in any medium, provided the original work is properly cited, the use is non-commercial and no modifications or adaptations are made. 